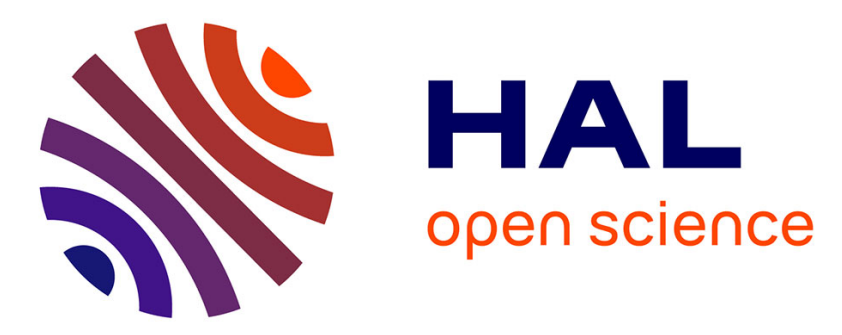

\title{
Structuration et scénarisation de documents pédagogiques numériques dans une logique de massification
}

Stéphane Crozat, Philippe Trigano

\section{- To cite this version:}

Stéphane Crozat, Philippe Trigano. Structuration et scénarisation de documents pédagogiques numériques dans une logique de massification. Sciences et Techniques Educatives, 2002, 9 (3-4). edutice-00000386

\section{HAL Id: edutice-00000386 \\ https://edutice.archives-ouvertes.fr/edutice-00000386}

Submitted on 5 Mar 2004

HAL is a multi-disciplinary open access archive for the deposit and dissemination of scientific research documents, whether they are published or not. The documents may come from teaching and research institutions in France or abroad, or from public or private research centers.
L'archive ouverte pluridisciplinaire HAL, est destinée au dépôt et à la diffusion de documents scientifiques de niveau recherche, publiés ou non, émanant des établissements d'enseignement et de recherche français ou étrangers, des laboratoires publics ou privés. 


\title{
Structuration et scénarisation de documents pédagogiques numériques dans une logique de massification
}

\author{
Stéphane Crozat, Philippe Trigano \\ UMR CNRS 6599 HEUDIASYC \\ Université de Technologie de Compiègne \\ BP 20529 - 60205 Compiègne Cedex \\ Email :Stephane.Crozat@utc.fr,Philippe.Trigano@utc.fr
}

\begin{abstract}
RÉSUMÉ. La croissance spectaculaire du numérique (Internet en tête), combinée aux besoins nouveaux dans le domaine de la formation (professionnelle notamment) ont conduit à un développement considérable de la production de documents pédagogiques numériques. Notre travail s'inscrit au sein de cette problématique, afin d'élaborer un modèle de conception industrielle qui permette l'application d'un modèle documentaire générique, et l'intégration de modèles pédagogiques spécifiques, dans une perspective de massification. Ce modèle a été conçu à partir d'une approche théorique depuis l'état de l'art (séparation fond/forme, séparation contenu/scénarisation et association information/actions) et une approche empirique depuis nos expérimentations (XF01 et SCENARI). Le résultat de ce travail est le méta-modèle SP/UL qui propose une approche méthodologique et technologique afin de représenter logiquement l'information multimédia pédagogique (sous forme d'Unités Logiques), de distribuer l'information au sein de scénarii pédagogiques (sous forme de Schémas Pédagogiques), et de présenter l'information pour la manipuler (sous forme de Feuilles de Comportement).

ABSTRACT. In one hand digital documents are more en more used and in the other hand new needs are emerging in the field of institutional education and professional training. The consequence is a skyrocketing development of instructional digital documents. In this paper we submit an industrial design model that aims to conciliate a generic documentary metamodel along with specific pedagogical models, in a mass-production context. Our research is based on a theoretical approach from the state of the art study (content/form separation, content/scenario separation and content/actions association) and on a empirical approach from practical experiments (XF01 and SCENARI). The result of our work is PS/LU metamodel which enables a methodological and a technological framework in order to: logically represent multimedia and pedagogical information (as Logical-Units), deal information towards pedagogical scripts (as Pedagogical-Schemas) and present information so that it can be manipulated (as Interaction-Sheets).

MOTS-CLÉS : Support numérique, contexte pédagogique, conception, modèle, structuration, interactivité, scénarisation

KEY WORDS: Digital media, instructional context, design, model, structuring, interactivity, scripting
\end{abstract}

Signature de l'article : nom de la revue. Volume $X-n^{\circ} X / 2000$, pages 1 à $X$ 


\section{Introduction}

La formation se développe aujourd'hui en quantité et en variété : elle n'est plus seulement l'apanage des écoles et des universités, mais elle investit le lieu de travail, le temps libre, cessant d'être seulement cette étape préliminaire à l'entrée dans la vie active, pour devenir un processus continu tout au long de la vie. Or toute pratique étant ancrée dans une technique, la mutation actuelle d'un domaine aussi prégnant que les métiers de la formation ne saurait être épargnée par l'arrivée en force du numérique et son invasion de la vie courante, portée par la micro-informatique en général et Internet en particulier. Et les enjeux ne sont pas des moindres puisque les universités et les centres de formation se posent aujourd'hui la question d'Internet en tant que "moyen de renouveler une relation pédagogique mise à mal par les effectifs et l'hétérogénéité croissante des étudiants [REV 00]", tandis que les entreprises y voient une solution propre à diminuer les coûts de déplacement et d'hébergement associés à la formation en entreprise [PEC 00].

Le numérique n'est ni une fatalité ni un miracle, mais simplement une technique nouvelle de manipulation de la connaissance qui vient s'intégrer progressivement aux autres techniques pré-existantes pour apporter les innovations qu'elle porte. " $L a$ combinaison des différents supports a toujours existé : l'oral n'a pas disparu avec l'écrit, ni le manuscrit avec l'imprimerie, ni le journal avec la télévision, ni le courrier avec le téléphone, etc. En revanche chacun s'est spécifié, sa sphère d'action a été redéfinie, parfois après un temps long et suscitant toujours des craintes de disparition [BOU 00]". La question qui se pose aujourd'hui aux acteurs de la pédagogie et de l'informatique, n'est donc pas celle de l'opportunité ou non d'introduire le numérique dans la pédagogie (il s'intègre de fait et sans besoin d'en décider) mais plutôt celle de comment l'appréhender et le maîtriser pour l'irriguer dans la meilleure direction. La réponse à cette question n'est pas dans une vision techniciste qui imposerait ses lois à la pratique, mais plutôt dans la recherche d'un couplage entre la pratique et la technique, mutuellement constituantes, afin de faire plier la technique aux besoins réels d'une part et de ré-interroger la pratique sur ses besoins d'autre part.

Si l'expérience dans le domaine des supports pédagogiques numériques est encore naissante, elle permet néanmoins de cadrer globalement les possibles offerts par ces technologies, encore nouvelles du point de vue des usages sinon du point de vue de la technique. Néanmoins les mutations récentes des métiers de la formation induisent une demande croissante qui se tourne naturellement vers les solutions du numérique, incontournables par les rêves qu'elles apportent et malgré les craintes qu'elles suscitent. Or les techniques actuelles de conception, encore globalement artisanales, ne sont pas en adéquation avec l'explosion de la demande et la massification qu'elle introduit. La rationalisation des coûts de conception, pour des environnements de formation de grande envergure, est un axe essentiel, dans la mesure où elle rend possible le maintien de la qualité malgré l'accroissement de la quantité. La prise en compte de la massification est un élément nécessaire, bien que 
non suffisant, pour permettre à la pratique de s'exprimer dans la technique et éviter qu'une technique mal adaptée ne la paupérise.

C'est dans ce cadre que nous plaçons notre travail, afin de rechercher des solutions conceptuelles et informatiques pour la conception de supports pédagogiques numériques qui soient à la fois en adéquation avec les pratiques actuelles de la formation et à la fois en adéquation avec le contexte actuel de massification. Gil parle "d'une formation individualisée de masse [GIL 00]".

Dans la première partie, nous procèderons à l'explicitation de notre problématique, puis à un bref état de l'art, et à la description de l'approche adoptée. Nous répondrons à cette problématique dans les parties 2 et 3 , par le méta-modèle SP/UL. Dans les parties 4 et 5 nous présentons l'instrumentation technologique et méthodologique permettant la mise en œuvre de notre modèle de conception. Enfin dans les parties 6 et 7 nous présenterons deux applications ayant permis son expérimentation et sa validation.

\section{Problématique}

\subsection{La massification}

Des méthodes artisanales de conception, telles que celles que nous avions développées au cours de travaux antérieurs [CRO 99], [CRO 00], [HU 00], [HU 01a], [HU 01b] ne sont pas adaptées au développement d'applications pédagogiques de grande envergure, traitant des milliers de pages de documents pour des milliers d'apprenants. Il devient donc nécessaire, dans ce cas, de proposer d'autres modèles, complémentaires, permettant de proposer des solutions à grande échelle tout en conservant les acquis de l'informatique pédagogique. Notre propos n'est pas de vouloir substituer une approche à une autre, mais plutôt de penser des méthodes différentes pour des contextes différents, avec des visions plus génériques pour les grands volumes et plus spécifiques pour les applications très contextuelles.

\begin{tabular}{|c|c|c|c|c|}
\hline Approche & Produit & Caractéristiques & Avantages & $\begin{array}{c}\text { Inconvénients } \\
\text { Artisanale }\end{array}$ \\
$\begin{array}{c}\text { CEuvre } \\
\text { unique }\end{array}$ & $\begin{array}{c}\text { Scénario spécifique } \\
\text { et contenus } \\
\text { spécifiques pour un } \\
\text { contexte spécifique }\end{array}$ & $\begin{array}{c}\text { Efficacité } \\
\text { optimum } \\
\text { Adaptation } \\
\text { manuelle } \\
\text { Maintenance }\end{array}$ \\
\hline Industrielle & $\begin{array}{c}\text { Système } \\
\text { documentaire }\end{array}$ & $\begin{array}{c}\text { Scénario générique } \\
\text { et contenus } \\
\text { génériques pour des } \\
\text { contextes } \\
\text { spécifiques }\end{array}$ & $\begin{array}{c}\text { Maintenance } \\
\text { Durée de vie } \\
\text { Multi-support } \\
\text { Réutilisation }\end{array}$ & $\begin{array}{c}\text { Investissement } \\
\text { initial }\end{array}$ \\
\hline
\end{tabular}

Tableau 1. Comparaison des approches artisanale et industrielle

L'enjeu de notre travail est la prise en compte de la massification dans la conception des supports pédagogiques numériques, à un niveau technologique et 
méthodologique, afin de contribuer à l'élaboration d'un modèle permettant de concilier la généricité (pour la rationalisation de la production et de l'édition), la spécificité (pour la contextualisation de la formation) et l'interactivité (pour l'efficience de l'apprentissage).

\subsection{Les acquis du domaine}

La recherche en EAO, à travers la mise au point de solutions technologiques exploitant les propriétés dynamiques et multi-média du support numérique, a montré son intérêt dans le cadre de la formation en instrumentant des concepts tels que la personnalisation, le guidage, l'autonomisation, la simulation, etc. Nous pourrions résumer cet intérêt par le fait que le caractère dynamique des logiciels facilite la mise en oeuvre de modèles évolués issus des travaux du XXème siècle en pédagogie, notamment le constructivisme. L'enjeu de notre recherche est de maintenir le niveau de ces acquis tout en abordant le problème de la massification. Notre étude de l'état de l'art, nous conduit à retenir un certain nombre de principes pour l'élaboration d'un modèle propre à y répondre :

- L'information numérique se caractérise par sa non linéarité qui implique des modes de représentation nouveaux [BAC 99]. Cette représentation est pertinente d'un point de vue pédagogique car elle permet d'adopter une pédagogie plus centrée sur l'activité de l'apprenant, et ainsi plus constructiviste et personnalisable. Les systèmes hypertextuels tels qu'ArchyMeDia [GUE 99] ou Nestor [ZEI 99] en sont l'illustration. Afin de gérer la désorientation issue de la non linéarité et de maintenir le sens d'un point de vue pédagogique, le recours à une scénarisation explicite permet à l'enseignant de cadrer les parcours suivis et les choix laissés à l'apprenant. L'automatisation partielle du déroulement de cette scénarisation, permet d'envisager des phases autonomes contrôlées par l'apprenant et des phases guidées contrôlées par l'enseignant via une programmation préalable. On citera par exemple les projets SMART-Learning [AJH 00] et SDM [GAS 98].

- La séparation entre structure logique et physique des contenus, permet d'envisager l'interopérabilité et la durabilité des contenus, deux conditions fondamentales pour le traitement de grands volumes. Le projet PolyTeX [BAC 98] en était un exemple intéressant fondé alors sur LaTeX, mais l'émergence du standard XML permet aujourd'hui la naissance de projet plus ambitieux tels EduML [BOU 00]. Couplée à une mise en oeuvre pertinente de l'indexation, à l'aide de métadonnées standardisées, permettant la localisation de l'information, et à un découpage fin en unités sémantiques relativement décontextualisées, cette approche permet également d'envisager la réutilisabilité des contenus produits dans des contextes matériels et organisationnels multiples. Afin de traiter ce problème de nombreuses organisations ont cherché à mettre au point un système de description standard de tout document pédagogique numérique à l'aide d'un ensemble de méta-données, nous citerons notamment la LOM des IEEE (grouper.ieee.org/groups/ltsc), le projet 
SCORM (www.adlnet.org) et le standard en construction JTC1/SC36 de l'ISO (tclsc36.org) qui a pour objet de fédérer les diverses initiatives.

- Si les logiciels auteurs s'inscrivent dans une perspective artisanale peu compatible avec la conception de grande masse de contenus, ils nous enseignent un élément essentiel de la conception : la production des contenus et des scénarii doit être dans les mains des auteurs et des enseignants (et non dans celles des informaticiens). Cette approche est la seule rationnelle pour envisager la production de contenus de qualité maîtrisés par les experts de ce contenu et de son enseignement. L'approche du CRIPT [CHO 00] est de ce fait très intéressante. Les solutions intermédiaires, tels que SERPOLET (www.a6.com) ou MALTED (www.malted.com) proposent un compromis entre la liberté de conception et la systématisation du traitement. Les travaux concernant l'ergonomie des logiciels pédagogiques [BAS 97], [MED 90], [ISA 00] montrent également le rôle fondamental que joue l'interface, partie visible de l'iceberg, dans l'acceptabilité et l'utilisabilité des produits. Une approche de conception se doit donc d'intégrer ce paramètre et d'assurer un niveau de qualité suffisant quant à la présentation des contenus et des fonctions de manipulation de ces contenus. Cette qualité doit pouvoir être assurée a priori par la logique de conception mise en oeuvre, ce qui est possible dans un cadre technologique basé sur des moteurs d'édition automatique de contenus structurés logiquement.

Si l'état de l'art nous fournit tous les éléments pour mettre en oeuvre une approche de conception propre à intégrer la massification, aucun système ne répond complètement à ce besoin. En effet les logiques mises en œuvre sont soient très orientées documentaire permettant de gérer la massification mais avec des possibilités de contextualisation restreinte, soit très orientées pédagogie et donc adaptées à un contexte mais difficile à exploiter en dehors. Le système ARIADNE [FOR 97] en est certainement aujourd'hui le plus proche, avec néanmoins la nécessité d'intégrer une approche de production assurant la maîtrise de la structure des contenus, ce qui passe selon nous par l'adoption de la structuration logique d'une part et la distinction claire entre les contenus et les scénarii d'autre part.

La raison de la difficulté à concilier documentaire et pédagogie vient principalement du fait que l'ingénierie documentaire sait résoudre le problème de la structuration logique de l'information et de sa mise en forme statique, alors que l'ingénierie pédagogique est avant tout attachée à la réalisation d'interfaces dynamiques. Les apports de l'informatique documentaire sont donc en l'état insuffisants pour répondre aux attentes de l'informatique pédagogique. Il nous est alors nécessaire d'envisager des solutions innovantes, permettant d'étendre la logique de généricité proposée par le documentaire à la l'édition automatique d'interfaces homme-machine répondant aux exigences de la pédagogie (i.e. interactives, permettant la navigation dynamique du contenu, la production de contenu par les apprenants, etc.). 


\subsection{Le couplage documentaire/pédagogique}

L'informatique documentaire est le modèle dominant pour la gestion de grands volumes d'information, ayant fait ses preuves dans d'autres domaines que la formation (la documentation technique industrielle par exemple). Ce modèle se fonde sur une représentation de l'information sous formes de documents, c'est à dire d'information non formalisée au sens de la logique, et donc lisibles par l'homme mais pas par la machine. L'intérêt de l'informatique étant la manipulation de l'information par le calcul, et cette manipulation ne pouvant se faire à partir de l'information représentée sémiotiquement dans les documents, il est nécessaire de structurer les documents au sein d'un modèle qui permet leur contrôle. Une telle approche peut-être résumée succinctement par un modèle à deux couches : une couche de discrétisation et de décontextualisation de l'information en unités documentaires modulaires et une couche de représentation formelle de ces unités de façon à les contrôler et les recontextualiser pour une pratique. En effet le support numérique invite à de nouvelles manipulations de l'information, ouvrant ainsi un nouveau champ du possible, mais dont toutes les combinaisons ne sont pas pertinentes. Il est donc nécessaire d'inscrire le support et son usage dans la pratique, qui seule dispose des fondements et de l'expérience pour contrôler les processus de manipulation de l'information et assurer le maintien du sens. La construction d'un modèle documentaire ad hoc pour une pratique de formation, n'a de sens selon nous que pensé conjointement avec une ingénierie pédagogique.

Il n'est pas raisonnable d'envisager que tout ce qui compose la pédagogie puisse être modélisé, car la pédagogie est à l'échelle de la complexité humaine et qu'une machine ne peut la maîtriser. Néanmoins une partie de la pratique pédagogique est fondée sur l'élaboration de scénarii d'apprentissages pour contrôler le processus d'apprentissage. Or l'essence de l'informatique étant le contrôle des processus dynamiques, la représentation de scenarii pédagogiques au sein d'une machine nous semble une hypothèse viable. Cette représentation nous fournit la couche formelle permettant le contrôle automatique de la manipulation dynamique des documents par la machine. Elle permet d'envisager la recontextualisation de contenus génériques pour leur exploitation au sein de parcours spécifiques. La recontextualisation assure l'inscription de la manipulation du support dans les usages et restreint cette manipulation aux possibles ayant du sens pour l'apprentissage.

La question que nous nous proposons de traiter est donc celle de l'élaboration d'un modèle de conception de supports numériques permettant la combinaison d'une logique documentaire et d'une logique pédagogique dans un cadre de massification. 


\section{Quelques principes fondateurs}

\subsection{Séparation fond/forme}

Le support numérique introduit une notion nouvelle dans la mesure où la façon dont l'information est inscrite (codée sous forme binaire) diffère de la façon dont elle est présentée (des caractères sur un écran par exemple). Le calcul effectué par l'ordinateur transforme la représentation interne de l'information en une présentation extériorisée sur un support pour le lecteur. Il est donc impossible sur un support numérique de faire coïncider l'inscription de l'information avec sa présentation. Il devient par contre possible de représenter une information indépendamment de sa présentation sous une forme donnée. Par exemple, si l'on veut présenter une même phrase sur un support papier pour la lecture et sur un support oral pour l'écoute, il est nécessaire de procéder à deux inscriptions, une écriture et un enregistrement. Avec le support numérique il est possible de ne procéder qu'à une seule inscription de l'information et à deux calculs différents pour obtenir deux présentations différentes de la même représentation.

$\mathrm{Si}$ le support numérique ouvre une possibilité d'indépendance entre représentation et présentation, il ne l'assure pas. En effet si l'on représente une information sous une forme qui n'a de sens que pour un support de présentation donné, il ne sera pas possible d'effectuer de calcul permettant de présenter cette information sur un autre support. Afin de disposer de cette possibilité, il est nécessaire de représenter logiquement l'information et non physiquement.

On appelle structuration logique de l'information une représentation explicitant la structure de l'information en fonction de ses caractéristiques intrinsèques et non en fonction des propriétés de présentation d'un support. On appelle structuration physique d'une information une représentation décrivant la façon dont une information doit être présentée sur un support donné. La structuration logique est associée au fond de l'information, tandis que la structuration physique est associée à sa forme sur un support. Notons qu'il est possible de calculer une ou plusieurs structurations physiques pour une même structuration logique. Il est possible de calculer d'autant plus de structurations physiques que la structuration logique est indépendante de ses supports de présentation.

L'enjeu de notre modèle étant de parvenir à mettre en place une approche de conception propre à répondre à un contexte de massification, la séparation entre structure logique et physique permet d'accéder à certaines propriétés telles que :

- La pérennité des contenus : en effet en étant dissociées d'un format physique, les informations sont indépendantes de l'évolution de ces formats. Il n'est donc pas nécessaire de reproduire régulièrement les contenus pour être compatible avec les différentes versions de formats, HTML ou Microsoft Word par exemple.

- La maintenance des contenus : la représentation logique ne nécessite pas le recours à des experts d'un langage de présentation, comme c'est le cas pour HTML 
par exemple, puisque le langage de représentation logique est défini par les experts du domaine considéré en fonction de la terminologie qui leur est propre.

- La portabilité des contenus : l'investissement consenti pour la production des contenus peut-être rentabilisé en dehors d'un système technique donné, puisque des contenus structurés logiquement sont par définition interopérables. Par exemple l'information peut être diffusée sur des supports multiples (papier, Internet, cd-rom, etc.) ou sur des environnements matériels multiples (systèmes d'exploitation, taille d'écran, capacité de réseau, etc.).

- La malléabilité des contenus : la structure des contenus étant connue, il est possible de manipuler cette structure et ainsi faire coïncider la présentation à des contextes d'usage différents. Par exemple on pourra proposer une vision synthétique de l'information (en en sélectionnant les éléments essentiels) ou au contraire une vision exhaustive.

- La séparation des métiers : La séparation entre structure logique et physique permet également la séparation entre les métiers d'auteur (la production de l'information logique) et les métiers d'éditeur (la mise en forme de cette information pour un support). Cette séparation permet d'augmenter les volumes de production et de réduire les coûts, puisque qu'elle ne nécessite plus le recours à des experts pluridisciplinaires capables de maîtriser à la fois le contenu et son édition.

\subsection{Séparation scénario/contenu}

La logique de conception documentaire conduit à distinguer les documents d'une part, formes sémiotiques d'appropriation de l'information discrétisée, et le contrôle de ces documents d'autre part, c'est à dire la dynamique suivant laquelle ils sont recomposés et présentés. Dans le cadre des applications pédagogiques, nous sommes ainsi amenés à distinguer les contenus de leur scénarisation. Cette distinction se révèle le second atout majeur (avec la séparation fond/forme) pour une gestion de la massification. En effet, elle nous permet d'envisager la possibilité de réutiliser des contenus génériques (i.e. valables pour une catégorie de contextes et non pour un seul en particulier) dans le cadre de scénarii spécifiques, rationalisant ainsi les coûts de production des contenus en leur offrant des contextes d'utilisation multiples, sans perte d'efficience puisque la re-scénarisation permet la re-contextualisation. De façon complémentaire on peut envisager la conception de scénarii complexes, expression d'une pédagogie donnée, et le réinvestissement de ces scenarii pour différents contenus. Enfin la séparation explicite entre l'espace sémiotique et l'espace de calcul permet de profiter de la dynamique du calcul propre au support, et ainsi automatiser (au moins partiellement) la gestion des processus pédagogiques (i.e. guider un apprenant dans un parcours pédagogique non linéaire en fonction de son activité).

Les scénarii étant fondés sur le calcul ils ne sont pas linéaires (séquence temporelle fixe), mais bien dynamiques (séquence dont la linéarité est construite en 
fonction de l'action de l'utilisateur). Considérant le calcul d'un scénario comme le fait de fixer un parcours dans un graphe de documents, l'unité de calcul est le document. Or étant donné la non-linéarité a priori de ce graphe, il est difficile, voire impossible, de faire des hypothèses valides sur les antécédents et les successeurs d'un document donné. Sur un support linéaire, par exemple un livre, il est facile de faire de telles hypothèses. Ainsi on pose que lors de la lecture de la page $\mathrm{N}$, la page $\mathrm{N}-1$ a déjà été lue et que la page $\mathrm{N}+1$ sera lue ensuite. Mais lors de la lecture d'un nœud du graphe, on ne peut présupposer des nœuds lus précédemment et des nœuds qui seront lus ensuite. Une solution générale à ce problème est le recours à une unité d'information correspondant à l'unité de calcul. On entend par unité d'information un document, nœud du graphe, dont la lecture est nécessaire et suffisante pour la compréhension d'un concept. Cette unité peut également être appelée grain autonome [BAC 98] ou unité sémantique [BAL 96] et se caractérise par une taille suffisamment petite pour acquérir une certaine autonomie vis à vis de son contexte : "plus les documents sont petits, plus ils sont réutilisables, car ils incorporent moins de contexte pédagogique", traduit depuis [FOR 99].

L'autonomie sémantique des unités de calcul est un enjeu complexe. Cette autonomie est impossible à atteindre de façon systématique et absolue, car cela supposerait la possibilité d'exprimer de l'information de façon générique en dehors de tout contexte. Bachimont parle de "grains autonomes mais pas indépendants [BAC 98]" pour exprimer cette limite. Balpe pose même la question de savoir si une trop grande autonomie est souhaitable dans la mesure où une trop grande fermeture sémantique conduit à une perte de sens générale [BAL 96]. A l'inverse une trop faible autonomie des unités d'information conduit à l'impossibilité de calculer des parcours dynamiques sensés et restreint les scénarii à une unique linéarité programmée. "Le principe est donc de proposer des unités de lecture renvoyant à des navigations possibles [BAC 98]", c'est à dire d'une part de tendre vers une certaine autonomie des unités d'information, relativement à un contexte, et d'autre part de proposer des scénarii de lecture tels qu'ils construisent nécessairement des lectures sensées par la présélection des arcs pertinents du graphe pour un contexte d'apprentissage donné (par opposition à une approche trop libre de la navigation qui autorise une grande combinatoire de parcours possibles).

\subsection{Association information/action}

Le couplage d'une ingénierie pédagogique avec l'ingénierie documentaire que nous avons choisi, nous conduit à nous interroger sur la problématique de réappropriation. Les notions de pédagogie active issues du mouvement constructivisme [PIA 67] et les notions de synthèse et réécriture issues de la théorie du support [STI 94], [BAC 96] nous montrent en effet que la compréhension, et donc l'apprentissage, ne peuvent se limiter à la lecture, au sens passif, d'information. Nous posons donc que toute information est associée à des activités de réécriture à 
mettre en oeuvre par le lecteur. Ces activités impliquent dans un cadre d'apprentissage la production effective et active d'informations nouvelles par l'apprenant, telles que des annotations, des résolutions d'exercices, des synthèses, etc.

La nature de ces activités est prescrite à la fois par la pratique, pédagogique en ce qui nous concerne (un cours peut être annoté, synthétisé, un exercice peut se résoudre, etc.) et par la nature du support (comment annoter sur un support numérique ?) et les possibilités nouvelles qu'il offre (l'exécution de simulations dynamiques par exemple est une nouvelle forme d'activité pour l'apprenant). Notre modèle se doit d'intégrer au delà de l'information et de sa scénarisation, les actions de réappropriation qui accompagnent ces informations.

L'information devant être associée à des activités de réappropriation, se pose alors la question de l'interface homme-machine dans le cadre du numérique et de la consultation des informations sur écran. La conception des interfaces est aujourd'hui un domaine bien maîtrisé, que nous avons étudié au cours de nos travaux antérieurs [CRO 99]. Dans le cadre de notre approche il n'est pas possible d'envisager que la réalisation de l'interface soit menée artisanalement pour chaque unité d'information. En effet la généricité acquise au niveau de la représentation serait sans intérêt sans généricité au niveau de la présentation. Cette généricité existe déjà dans le cadre de l'ingénierie documentaire, par l'intermédiaire de la notion de feuille de style, qui permet d'associer une représentation physique à une représentation logique, et permet ainsi le traitement automatique de la présentation des informations logiquement structurées indépendamment de leur volume. Néanmoins, si les feuilles de style traitent le problème de la présentation statique pour la lecture, elles ne traitent pas la mise à disposition de fonctions dynamiques permettant l'action de l'apprenant sur l'information (la réécriture).

Notre modèle a donc cherché à intégrer une dimension fondamentale dans un cadre pédagogique et absente des modèles documentaires classiques, la notion de feuille de comportements, extension des feuilles de style, permettant de représenter les interactions de l'apprenant avec l'information et la gestion de ses productions personnelles selon une logique similaire. Cette extension relève d'un enjeu central dans la mesure où un modèle de structuration et de scénarisation des contenus sans un modèle d'édition automatique d'interfaces interactives est soit inefficient d'un point de vue documentaire (l'édition devant être manuelle in fine, n'exploitant alors plus les potentiels de la représentation logique), soit inefficient d'un point de vue pédagogique (l'édition automatique conduisant à des interfaces statiques, n'exploitant plus les potentiels du support dynamique). 


\section{Le méta-modèle SP/UL}

Le méta-modèle $\mathrm{SP} / \mathrm{UL}$ est la réponse conceptuelle à notre problématique, intégrant les caractéristiques de séparation fond/forme, contenu/scénari et l'association information/action.

\subsection{Unité Logique}

On définit une unité logique (UL) comme une agrégation de contenus mobilisables dans un cadre d'apprentissage, le terme contenu désignant toute forme sémiotique participant à l'explication d'un concept, tel que :

- Une UL est identifiée par un identifiant unique et un titre, permettant respectivement sa localisation par une machine et par un homme.

- Une UL est multimédia, c'est à dire que les contenus monomédias qui la composent mobilisent potentiellement des formes sémiotiques différentes.

- Une UL est structurée logiquement, c'est à dire qu'elle assure une représentation logique de l'information le moins liée possible à une représentation physique, tout en optant pour des formats standards lorsque le représentation physique est inévitable (cas d'une image par exemple).

- Une UL est méta-informée, c'est à dire qu'elle est associée à une entête décrivant sa nature pédagogique (objectifs, temps de travail, niveau, etc.). Une entête est définie par un ensemble d'attributs ayant des valeurs fonctions de la nature de l'unité. Les contenus permettent donc de réaliser les spécifications pédagogiques décrites par l'entête. Par exemple le Dublin Core définit une entête pour les documents en général, la LOM définit une entête pour les documents pédagogiques.

- Une UL est active, c'est à dire que l'on peut spécifier pour chaque unité un ensemble d'activités de réappropriation associées au contenu favorisant l'assimilation. L'apprentissage ne se limitant pas à la lecture, il suppose de la part de l'apprenant une réécriture active des contenus lus (annotation du cours, résolution d'exercices, etc.)

- Une UL est dynamique, c'est à dire qu'elle autorise l'enregistrement des informations produites par l'apprenant lors de l'exécution des actions associées à l'unité. En effet, les activités de réécriture impliquent que l'apprenant produise de l'information, relativement au contenu, au cours de son apprentissage. Une unité dynamique est donc composée d'une partie statique, qui est définie lors de sa conception par les auteurs, et d'une partie dynamique qui est produite par les utilisateurs au fur et à mesure de leur apprentissage.

- Une UL est évaluable, c'est à dire qu'elle peut-être associée à une fonction portant sur les valeurs de ses actions associées. En effet, les actions associées aux unités donnent lieu à des productions, qui elles-mêmes peuvent donner lieu à des 
évaluations, soit par l'apprenant lui-même, soit par le tuteur, soir automatiquement par le système.

- Une UL tend à être suffisante pour sa compréhension, c'est à dire qu'elle n'a pas besoin de renvoyer de façon explicite à d'autres unités pour être lue et que l'exécution correcte par l'apprenant des actions qui lui sont associées suffit à sa réappropriation (une exécution correcte correspondant à une production évaluée comme juste par un tuteur humain ou par un programme informatique). Cela implique que l'unité peut-être lue pour elle-même et qu'elle est autonome dans un contexte (sous réserve que le lecteur dispose des pré-requis nécessaires, par exemple).

- Une UL tend à être nécessaire pour sa compréhension, c'est à dire qu'elle doit être lue dans son ensemble et que l'ensemble de ses actions associées doit être exécuté correctement pour être comprise. Cela implique que l'unité est minimum et insécable et donc qu'aucune partie extraite et isolée de l'unité n'a de sens indépendamment $\mathrm{du}$ tout (c'est à dire qu'aucune sous partie de l'unité n'est suffisante).

- Une UL est valide par rapport à un modèle de référence qui décrit sa structure logique et sa matérialisation sur un support via une feuille de comportement.

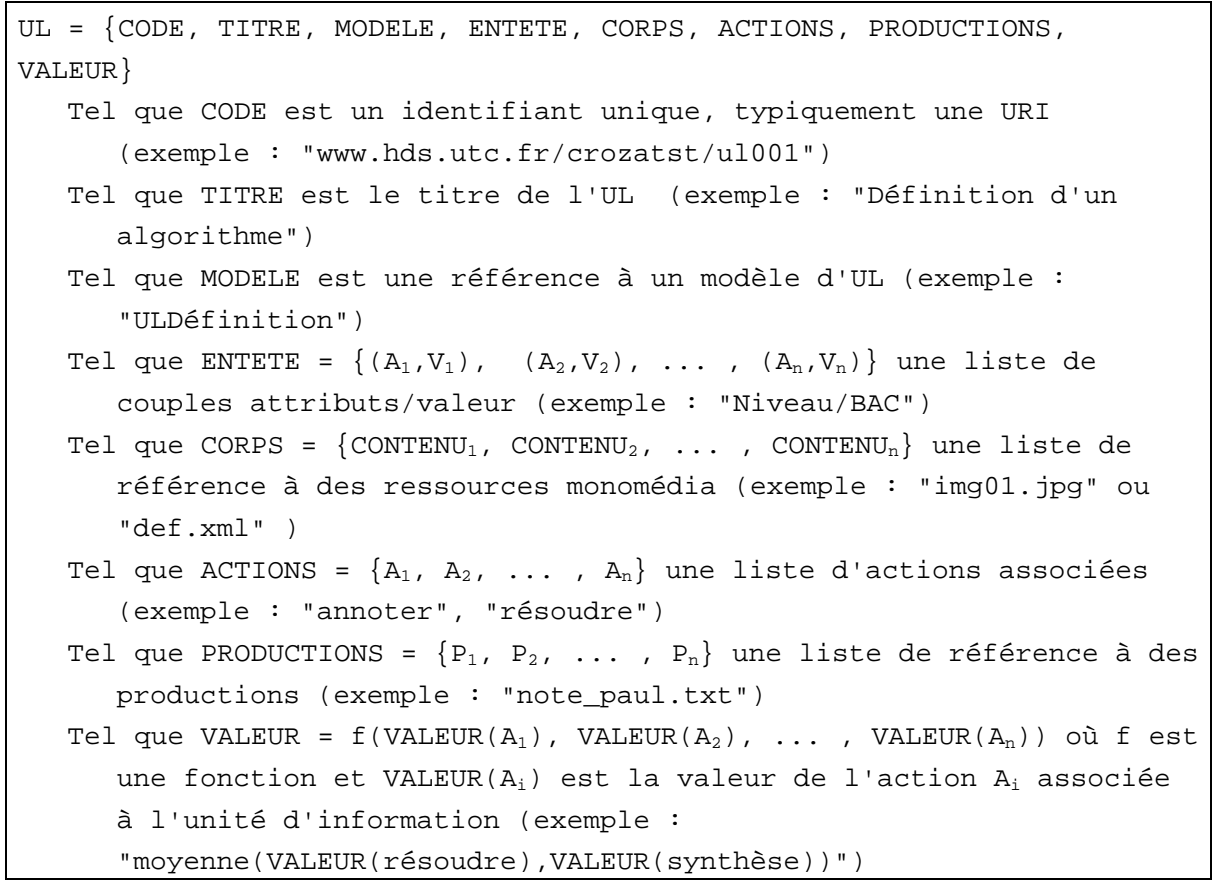

Figure 1 : Notation d'une Unité Logique 


\subsection{Schéma Pédagogique}

On définit un schéma pédagogique (SP) comme une description d'un ou plusieurs parcours possibles d'un ensemble d'UL par un graphe représentant des relations de précédence entre ces UL, tel que :

- Un SP est "raisonnablement linéaire", c'est à dire que tous les parcours qu'il décrit ont une même et unique unité d'information d'entrée et une même et unique unité d'information de sortie, et que le nombre de parcours possibles est de l'ordre de $\mathrm{n}$, avec $\mathrm{n}$ le nombre d'UL scénarisées (tandis que le nombre total de parcours possibles est de l'ordre de $n$ !). Remarquons dans ce cas qu'un SP représentant des relations de précédence entre des SP raisonnablement linéaires est aussi un SP raisonnablement linéaire. En effet si l'on remplace chaque SP fils par son graphe d'UL, et si l'on relie son UL de sortie avec les UL d'entrée des SP fils le suivant, on obtient bien un graphe de description d'UL respectant la définition ci-dessus.

- Un SP est transitionnel, c'est à dire que les relations de précédence entre les unités d'information sont informées par un texte décrivant la transition entre les SP ou UL d'entrée et les SP ou UL de sortie.

- Un SP est méta-informée (suivant la même définition que pour une UL).

- Un SP est actif (suivant la même définition que pour une UL).

- Un SP est dynamique (suivant la même définition que pour une UL).

- Un SP est évaluable, c'est à dire qu'il peut-être associé à une fonction portant sur les valeurs de ses actions associées et/ou sur les valeur de ses SP ou UL scénarisés.

- Un SP est contraint, c'est à dire que le passage d'un nœud à un autre au sein du scénario qu'il décrit est soumis à une condition, qui est alors une fonction booléenne portant sur les valeurs des entrées.

- Un SP tend à être suffisant (suivant la même définition que pour une UL).

- Un SP tend à être non nécessaire, c'est à dire que les UL ou SP qu'il scénarise sont au maximum suffisants (des UL suffisantes impliquant des SP sécables).

- Une SP est valide par rapport à un modèle de référence qui décrit sa structure et son comportement.

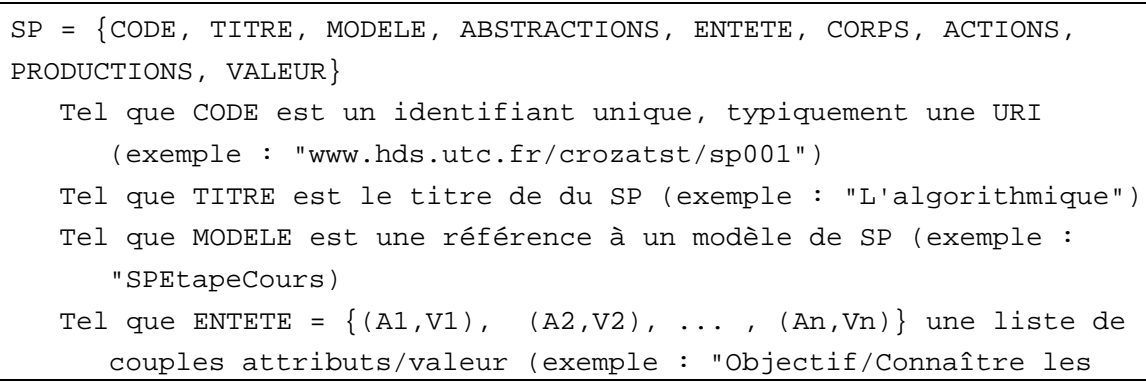




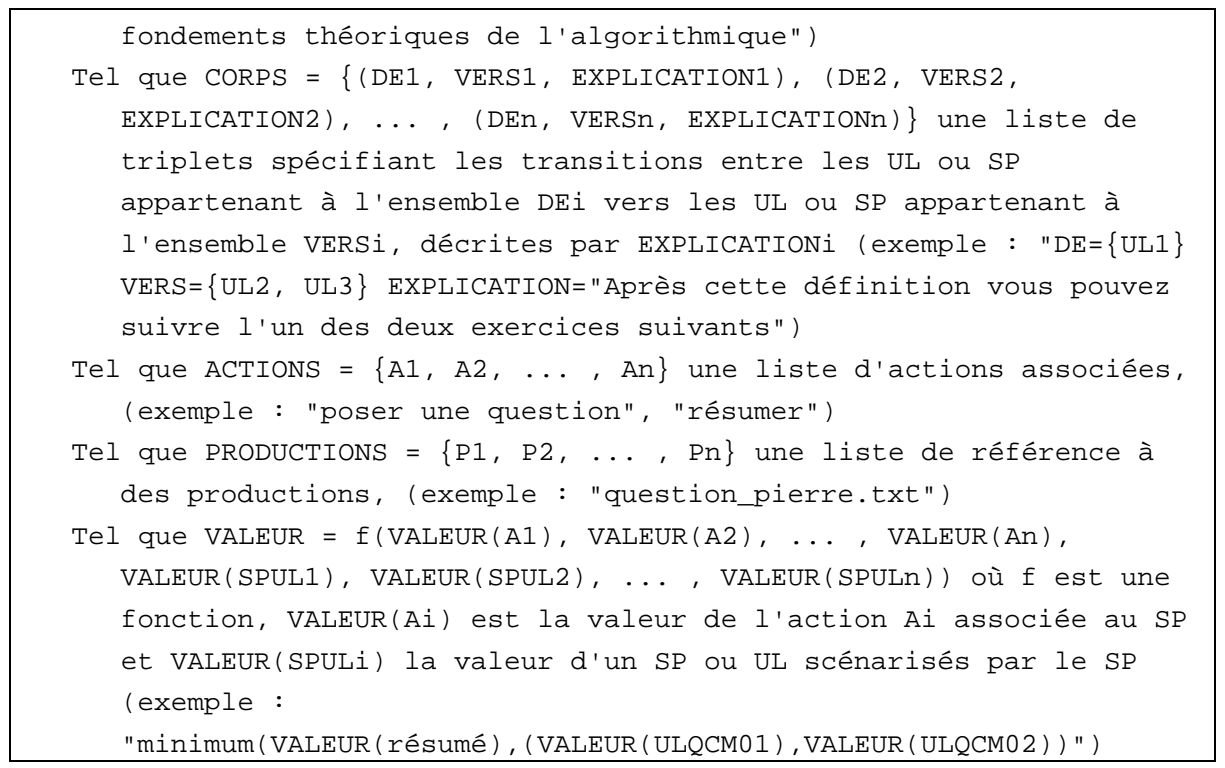

Figure 2. Notation d'un Schéma Pédagogique

\subsection{Modèle}

La notion de modèle permet d'associer modèle structurel et d'activité, et fournir ainsi le cadre formel pour la spécification du contexte pédagogique. La notion de modélisation s'inscrit au croisement de l'ingénierie documentaire et de l'ingénierie pédagogique propres à notre méta-modèle.

Les formats pédagogiques liés au contexte d'application de la formation permettent de définir a priori un ensemble de règles structurelles, formant des modèles capables de prédéfinir la structure logique des UL ou des SP. Une UL pour être représentée logiquement et non physiquement, implique que les contenus qui la composent soient structurés en fonction du type d'information et de leur rôle pédagogique (définition, exemple, explication, etc.). Dans un contexte pédagogique donné on cherche à définir une typologie d'UL répondant de façon pertinente aux spécificités du contexte pédagogique. De la même façon un SP décrit la structure d'enchainement d'UL ou de SP et s'il est possible d'imaginer une combinatoire très élevée de scénarii différents pour un même ensemble de contenus, il n'est pédagogiquement sensé que de mobiliser certains schémas types répondant à une logique pédagogique.

Les formats pédagogiques d'un contexte permettent également de définir l'ensemble des actions de réappropriation associées à un type de contenu ou de scénario. Il est en effet plus raisonnable de définir les actions de réappropriation au 
niveau d'un modèle plutôt qu'au niveau de chaque instance. Ainsi, par exemple, deux exercices de même type seront toujours associés aux mêmes activités, puisque pédagogiquement, ils relèvent du même format.

On définit un modèle de SP ou d'UL comme la combinaison d'un modèle structurel définissant a priori leur structure logique et d'un modèle d'activité définissant a priori leurs actions de réappropriation. Un modèle définit alors la nature pédagogique de l'unité indépendamment de son contenu et procède à l'instanciation du méta-modèle dans un contexte.

\subsection{Feuille de Comportement}

La représentation logique de l'information suppose l'association de cette représentation à une présentation physique permettant sa lecture et sa manipulation sur un ou plusieurs supports. Sur un support numérique cette représentation consiste en un affichage de l'information, mais également en la matérialisation des actions associées aux unités dynamiques et en l'interprétation du formalisme de SP pour exécuter les scénarii pédagogiques. On appelle comportement une telle matérialisation dynamique d'une UL ou d'un SP sur un support numérique. La notion de comportement inclut notamment l'affichage des différentes ressources, offrant une ergonomie de lecture adaptée aux types de média mobilisés, le déroulement des scénarii, incluant le gestion des transitions et des conditions, une interface hommemachine permettant l'exécution des actions, les mécanismes de stockage et de restitution des productions de l'apprenant, etc. Plutôt que de chercher à spécifier un comportement particulier pour chaque unité, il est possible d'associer un comportement à chaque modèle, et ainsi disposer de comportements communs pour chaque type d'unité. Cette hypothèse implique que toutes les unités valides par rapport à un même modèle se comporteront de façon identique.

On définit une Feuille de Comportement (FC) comme la spécification d'une matérialisation physique, en terme de présentation et d'interaction, de l'ensemble des SP ou UL valides par rapport à un modèle pour un support donné. L'enjeu des feuilles de comportement est à la fois conceptuel et technologique puisqu'il s'agit de répondre à deux questions : quel langage proposer aux éditeurs pour qu'il formulent leur besoins de mise en forme mais aussi et surtout d'interaction ? et quelle technologie mettre au point pour interpréter ce langage et réaliser automatiquement les interfaces homme-machine?

Notons qu'il peut exister plusieurs FC pour un même modèle, ce qui permet d'associer des représentations physiques différentes en fonction des environnements ou des supports. L'approche des modèles de comportement permet de passer d'une gestion artisanale à une gestion de masse. En effet la quantité de travail à fournir en terme de programmation informatique d'un support est proportionnelle au nombre de modèles différents qui existent et non au nombre d'unités. Cette approche implique 
de plus une standardisation et une homogénéisation qui permettent d'assurer à priori le comportement d'une unité. Il est de même possible d'avoir une approche multisupport (i.e. disposer de la même information sur des supports différents sans réécriture). Le quantité de travail est dans ce cas proportionnelle au nombre de supports multiplié par le nombre de modèles, mais reste indépendante du volume d'information considéré. Enfin, l'approche réduit dans les mêmes proportions les coûts de maintenance technologique puisqu'un changement du langage de matérialisation physique des unités équivaut au pire à une réécriture des feuilles de comportement, donc toujours proportionnelle aux nombre de modèles et non au volume de l'information. L'approche permet donc de traiter au moindre coût de larges volumes répondant à des modèles communs, mais est bien entendu sans intérêt si chaque unité doit avoir un comportement particulier.

Nous donnons ci-dessous un exemple d'association de comportements pour un modèle d'UL. La syntaxe d'une feuille de comportement étant évidemment définie en fonction d'une implémentation technologique, les comportements sont exprimés en langage informel.

\begin{tabular}{|c|c|c|}
\hline \multicolumn{2}{|c|}{ Eléments de l'UL } & Comportements \\
\hline \multirow{2}{*}{$\begin{array}{l}\text { Méta- } \\
\text { informations } \\
\text { Types }\end{array}$} & Mots-clés & Ignorer \\
\hline & Objectifs & $\begin{array}{l}\text { Afficher dans l'onglet secondaire et appliquer la XSL entete.xsl } \\
\text { pour la mise en forme }\end{array}$ \\
\hline \multirow{3}{*}{$\begin{array}{l}\text { Ressources } \\
\text { Types }\end{array}$} & $\begin{array}{l}\text { Définition } \\
\text { (texte XML) }\end{array}$ & $\begin{array}{l}\text { Afficher dans l'onglet principal et appliquer la XSL corps.xsl } \\
\text { pour la mise en forme }\end{array}$ \\
\hline & $\begin{array}{l}\text { Exemple } \\
\text { (Vidéo) }\end{array}$ & $\begin{array}{l}\text { Si "Question" et format("Question")="vidéo" alors ajouter une } \\
\text { commande de lancement de la séquence dans le menu de } \\
\text { gauche / Sinon afficher dans l'onglet principal et démarrer }\end{array}$ \\
\hline & $\begin{array}{l}\text { Question } \\
\text { (Vidéo ou } \\
\text { Son) }\end{array}$ & $\begin{array}{l}\text { Si format("Question")="audio" ou si "Exemple" alors ajouter une } \\
\text { commande de lancement de la séquence dans le menu de } \\
\text { gauche / Sinon afficher dans l'onglet principal et démarrer }\end{array}$ \\
\hline \multirow{2}{*}{ Actions } & Annoter & $\begin{array}{l}\text { Ajouter une commande dans l'onglet de gauche, sur action } \\
\text { afficher une fenêtre pop-up pour la saisie et mise en forme de la } \\
\text { fenêtre avec annoter.xsl }\end{array}$ \\
\hline & Répondre & $\begin{array}{l}\text { Si "Question" alors à la fin de l'exécution de Question afficher } \\
\text { une fenêtre pop-up pour la saisie et mise en forme de la fenêtre } \\
\text { avec répondre.xsl }\end{array}$ \\
\hline
\end{tabular}

Tableau 2. Exemple de feuille de comportement pour un modèle d'UL

\subsection{Nécessité et suffisance}

La propriété de suffisance pour une UL permet sa réutilisation dans des contextes différents de celui pour lequel elle a été produite. Par exemple la même définition du théorème de Thalès, si elle est suffisante, pourra être utilisée dans un cours de mathématiques et dans un cours de physique. De telles UL ont donc une valeur ajoutée forte, puisque l'investissement consenti pour leur production pourra être rentabilisé dans plusieurs formations différentes. La conséquence la plus 
importante dans notre cadre est l'indépendance entre les UL et les SP. En effet il est alors possible de re-scénariser des UL produites pour un premier scénario, et ainsi constituer plusieurs scénarii différents pour un même ensemble d'information. Cette possibilité permet d'envisager une personnalisation de la formation à grande échelle, en remodelant une formation pour un contexte spécifique, sans nouvel effort de production de contenus. La propriété de suffisance étendue aux SP facilite la possibilité de re-scénarisation. Il devient ainsi possible de re-scénariser une formation à un niveau micro (l'UL) ou bien à un niveau macro (le SP). La propriété de nécessité d'une UL permet quant à elle d'optimiser la re-scénarisation et la réutilisation, puisqu'elle assure le niveau de recombinaison le plus fin possible.

Précisons néanmoins que, la production d'UL se faisant toujours dans le contexte d'un scénario, il n'est pas raisonnable d'imaginer une pratique consistant à produire des UL indépendamment de tout usage pédagogique prédéfini. L'enjeu consiste donc à produire des UL nécessaires et suffisantes, afin qu'elles aient potentiellement un sens en dehors du scénario initial pour lequel elles ont été produites. Des UL nécessaires et suffisantes existent donc dans le contexte d'au moins un scénario, et également en tant que ressources décontextualisées, mobilisables dans un nouveau contexte. Ainsi la séparation rendue possible entre scénario et information ne signifie pas que l'information est disponible en dehors d'un scénario pédagogique ni qu'elle dispose d'une autonomie absolue, mais qu'elle est décontextualisable et recontextualisable au sein de plusieurs scénarii dans la limite d'un ensemble fini de contextes ayant des similarités fortes entre eux.

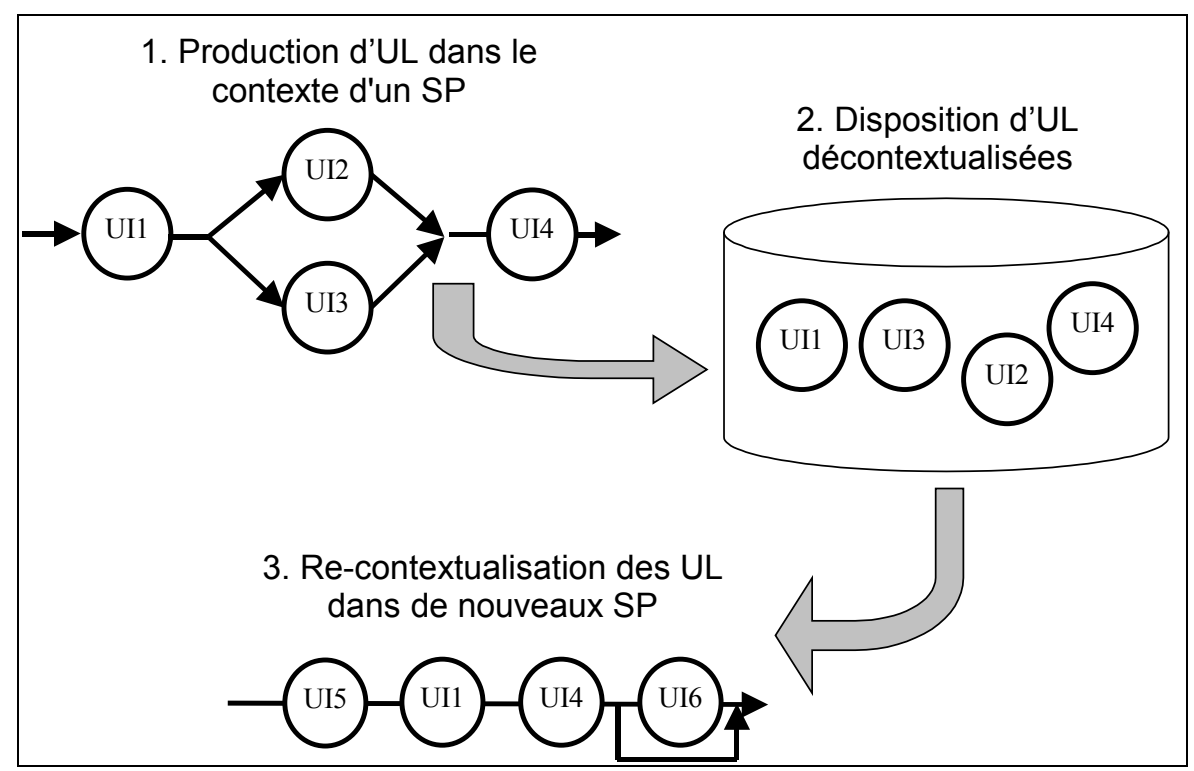

Figure 3. Mécanisme de réutilisation d'UL au sein de différents $S P$ 
Il est possible de faire une analogie avec le langage, en considérant que les phonèmes sont des ressources, sans sens propre, mais répondant à un standard, les UL des mots, qui n'ont du sens qu'en contexte mais peuvent être réutilisés dans plusieurs contextes différents, et les SP des phrases, qui donnent aux mots leur sens dans un contexte.

\subsection{Résumé du méta-modèle}

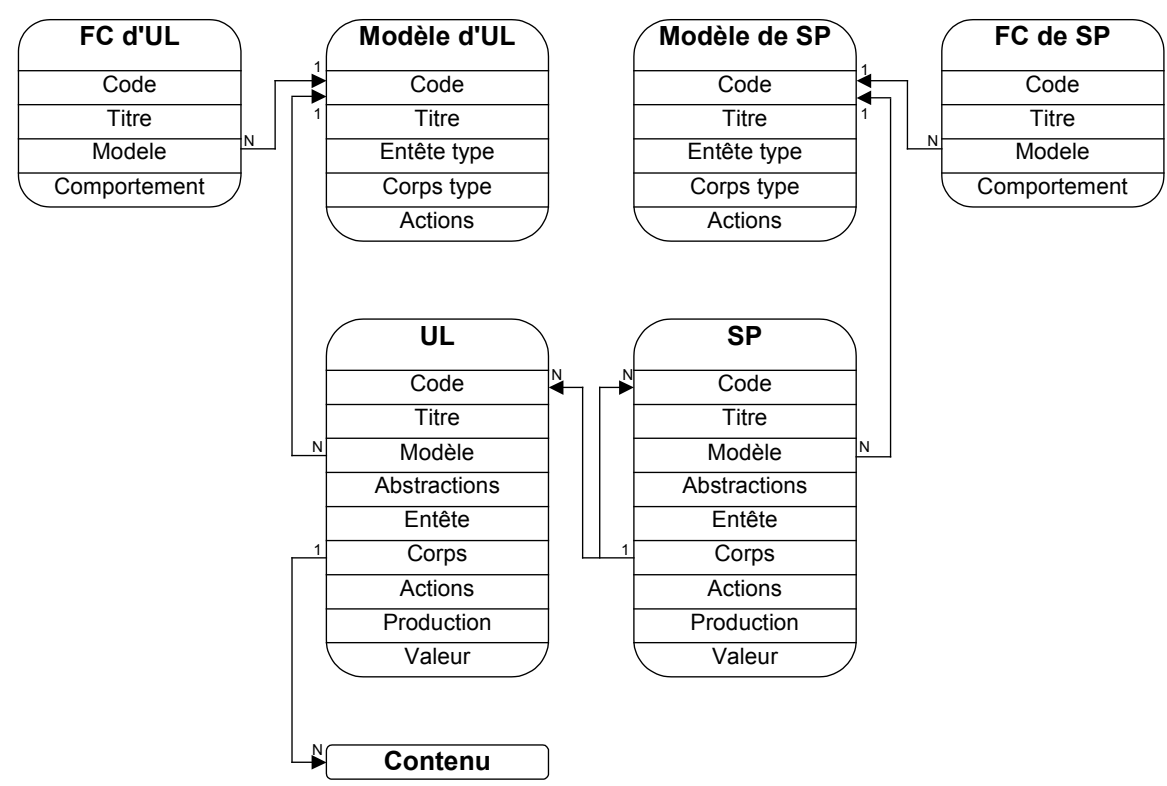

Figure 4. Représentation graphique du modèle $S P / U L$

\section{Conséquences technologiques}

Le méta-modèle SP/UL propose une réponse conceptuelle à la question de la massification, en se fondant sur la structuration et la scénarisation des contenus. Bien entendu cette approche ne peut être opérationnelle que couplée à une solution concrète d'informatisation, permettant sa réalisation. Les technologies XML s'inscrivent comme la solution actuellement la plus adéquate pour la mise en œuvre de notre approche documentaire, car elles répondent précisément à nos besoins, sont faciles d'usage et sont en pleine expansion dans la communauté informatique. Ces technologies permettent en effet de résoudre le problème de la structuration logique (par balisage des documents), de la modélisation a priori (par définition de types de document devant obéir à une certaine structure) et de la présentation des documents (par transformation des documents balisés en un format informatique de 
présentation). De plus ces technologies se caractérisent par une appréhension conceptuelle et technique très simple et par une manipulation très souple. Enfin XML est aujourd'hui un standard du W3C, déjà très largement utilisé, en plein croissance, et disposant d'outils mûrs pour sa mise en œuvre.

- Le méta-langage XML pour la représentation logique : L'eXtensible Markup Language est un méta-langage, c'est à dire un langage permettant de définir d'autre langages, proposé comme une recommandation par le W3C en 1998. La fonction d'XML est la représentation d'informations en se fondant sur la structuration logique du texte, par le repérage explicite de ses éléments constitutifs (le chapitre, le paragraphe, le mot important, etc.) et la hiérarchisation de ces éléments entre eux (un paragraphe appartient à un chapitre, un mot important à un paragraphe, etc.). XML ne se préoccupe pas de la présentation physique des informations (mise en page, polices de caractères, etc.). Afin de permettre cette structuration logique, XML procède par ajout de balises (des méta-informations structurantes) au contenu du texte, c'est à dire entre les mots constituant le texte.

- Le langage de DTD pour la modélisation structurelle : On peut contraindre la structure d'un document XML afin d'assurer a priori qu'elle respecte une forme particulière. On a pour cela recours à une Document Type Definition (DTD) qui permet de spécifier quels éléments un document peut mobiliser, et la hiérarchie que doivent respecter ces éléments. Une DTD permet donc de créer un langage XML particulier, propre à une classe de documents. Cette spécification se révèle un élément majeur, préalable à la constitution de tous documents XML, dans la mesure où c'est elle qui permet la standardisation des structures de documents, nécessaire pour l'interopérabilité ou les traitements automatiques des documents (pour en systématiser les présentations physiques par exemple). Un document XML respectant les règles structurelles imposées par une DTD est dit valide par rapport à cette DTD. Etablir le modèle structurel d'une UL équivaut alors à l'associer à une DTD. ${ }^{1}$

- Le langage XSL pour l'édition statique : XML nous permettant de représenter logiquement l'information, il est nécessaire, pour envisager son édition sur un support de réappropriation de disposer d'un mécanisme permettant de transformer cette représentation logique en représentation physique (HTML, PDF, etc.). L'eXtensible Style-sheet Language (XSL), nous fournit la solution technique à

${ }^{1}$ Le W3C propose aujourd'hui un nouveau formalisme pour représenter les structures de document, le XML Schema. Ce formalisme est plus puissant que le langage de DTD, il permet notamment le typage des données. De plus il présente l'avantage d'adopter une syntaxe XML alors que les DTD ont une syntaxe propre, ce qui permet une homogénéisation du cadre de travail et la possibilité de traiter les définitions de types de documents comme des documents XML (et donc par exemple de les générer ou transformer comme des documents XML). 
ce problème. XSL est en effet un langage permettant de transformer un document XML en un autre document de format quelconque. Le langage XSL est fondé sur des instructions permettant de sélectionner des éléments du document source XML et de générer les informations correspondantes sur le document cible. Par exemple XSL permettra d'écrire des instructions du type "si j'ai un élément <important>mot</important> dans la source alors je génère $\langle\mathrm{b}\rangle \operatorname{mot}\langle/ \mathrm{b}\rangle$ dans la cible". Une telle instruction permettra de mettre tous les mots repérés comme important dans le document XML en gras dans un format d'édition HTML. Un programme XSL est un langage déclaratif, récursif, à base de règles de type prémisse-conclusion ou la prémisse permet de sélectionner des nœuds de la source et la conclusion définit le texte à écrire sur la cible. XSL nous fournit donc la technologie pour gérer la transformation de documents logiques en documents physiques, en permettant la mise en oeuvre d'une édition multicontexte puisqu'il suffit d'écrire $\mathrm{n}$ programmes XSL et de les appliquer au même document XML pour disposer de $\mathrm{n}$ documents différents correspondant à différents choix de présentation, de supports, de formats, etc.

- Le langage de FC pour l'édition dynamique : Les feuilles de style répondent conceptuellement et technologiquement à la problématique d'édition à grande échelle pour un support statique de documents représentés logiquement. Elles restent néanmoins insuffisantes pour l'édition de documents numériques destinés à un support de lecture dynamique tel que l'ordinateur car elles se limitent à une logique de mise en forme et n'intègrent pas l'interaction entre l'utilisateur et l'information. Nous avons donc construit une couche supplémentaire, le langage de FC. Ce langage permet de développer des modèles de comportement exploitant le langage XSL pour la mise en forme des informations statiques (le contenu) et dynamiques (les productions) et permettant de spécifier les actions associées au modèle. Le langage comprend deux fonctions principales : la première permet d'associer le modèle à des comportements indépendants du contenu (par exemple l'action d'annotation) et de gérer leur mise en forme ; la seconde permet d'associer des éléments du contenu à des comportements dépendant du contenu (typiquement l'affichage d'information, ou bien la spécification d'actions telle que la réponse à une question de compréhension posée dans une UL). Le langage de FC est donc la dernière brique permettant d'implémenter le méta-modèle SP/UL, nous fournissant une approche documentaire, dans le prolongement du langage XSL, pour l'édition de documents interactifs à partir de contenus structurés logiquement.

Le schéma ci-dessous fournit donc un exemple typique d'environnement technologique propre à instrumenter la mise en place d'une logique de conception adapté au méta-modèle SP/UL : 


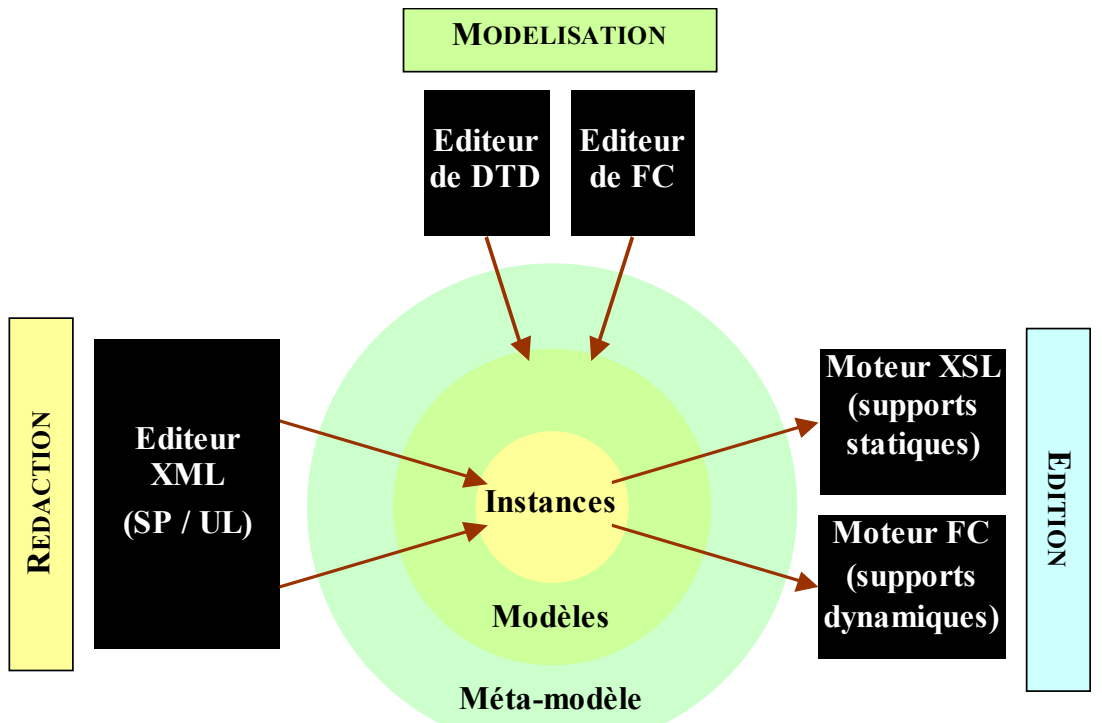

Figure 5. Environnement technologique pour l'implémentation du modèle SP/UL

\section{Conséquences méthodologiques}

Le méta-modèle SP/UL, permet de représenter des informations pédagogiques sur un support numérique dans une logique d'industrialisation, selon les principes de séparation de fond et forme, de séparation de contenus et de scénario et d'association d'actions de réappropriation aux informations. Il est nécessaire d'articuler ce modèle de conception avec une méthode permettant de mettre en oeuvre le modèle dans un cadre réel de conception massive. Cette méthode est inspirée du modèle de la chaîne éditoriale (analyse de contenus, modèle documentaire, rédaction, édition) mobilisée en ingénierie documentaire plutôt que d'une approche de génie logiciel classique (modèles en $\mathrm{V}$ ou Spirale par exemple, basés sur des séquences itératives de spécification, réalisation et validation). Elle permet de séparer les expertises impliquées dans la mise en oeuvre du modèle et d'articuler la chaîne de conception autour de six métiers : l'expertise pédagogique (pour contextualiser le méta-modèle), l'expertise du méta-modèle SP/UL (pour la modélisation), l'expertise auctoriale (pour la production du contenu), l'expertise de la rédaction (pour la mise en forme logique), l'expertise de l'édition (pour la mise en forme physique) et l'expertise du suivi (pour la gestion de la communication entre les autres acteurs).

- L'expertise pédagogique a pour rôle l'explicitation de l'intentionnalité pédagogique dans laquelle s'inscrit le support. En tant que spécification de l'ingénierie pédagogique en contexte, elle est la base indispensable à la contextualisation du modèle documentaire. Dans notre cadre son objet n'est pas de 
parvenir à une expertise exhaustive, mais de se limiter aux éléments nécessaires à l'instanciation du méta-modèle SP/UL dans le contexte considéré.

- L'expertise de modélisation a pour rôle, à partir des spécifications explicitées au cours de l'expertise pédagogique, de réaliser une instance du méta-modèle SP/UL pour le contexte particulier considéré. Elle permet donc de définir les modèles (structurels et d'activité) des SP et UL qui seront utilisés par les auteurs et les éditeurs.

- L'expertise auctoriale a pour rôle la production effective des contenus (UL) et scénarii (SP). Cette production peut se faire dans un cadre technologique quelconque, mais nécessite néanmoins de la part des auteurs une compréhension globale des différentes logiques liées au méta-modèle SP/UL afin d'adapter leur métier aux contraintes qu'il implique. Notons que cette expertise inclut la notion de maintenance (production à partir de l'existant) et d'indexation (production des métainformations).

- L'expertise rédactionnelle a pour rôle la mise en forme logique des SP et UL produites par les auteurs, en instanciant les modèles spécifiés par l'expertise de modélisation. Elle ne nécessite pas de compétences informatiques (au sens de la réalisation de programme) puisque la rédaction se fait dans le cadre d'un langage logique lié au contexte et non d'un langage artificiel de programmation.

- L'expertise éditoriale a pour rôle la réalisation des programmes permettant la transformation des contenus structurés logiquement en présentations physiques pour un usage. Dans le cas de l'édition papier elle consiste à écrire les feuilles de style, dans le cas de l'édition numérique elle consiste à écrire des feuilles de comportement. L'édition est réalisée à partir des modèles, et non des contenus. Il s'agit bien d'une édition générique pour l'instance pédagogique du méta-modèle et non d'une édition spécifique à chaque contenu. Outre une indépendance vis à vis du volume à traiter, permettant la massification, cette propriété assure que les éditeurs n'ont pas besoin d'être experts du contenu, il leur suffit de comprendre sa structure.

- L'expertise de suivi a pour rôle la gestion des mécanismes de rétroaction et d'ajustement entre les autres expertises. En effet, la séparation entre les métiers, que nous avons introduite pour favoriser une approche méthodique, ne peut être absolue et suppose un suivi global pour être mise en oeuvre.

Nous pouvons résumer le processus de conception selon ces six métiers sur le schéma ci-dessous. 


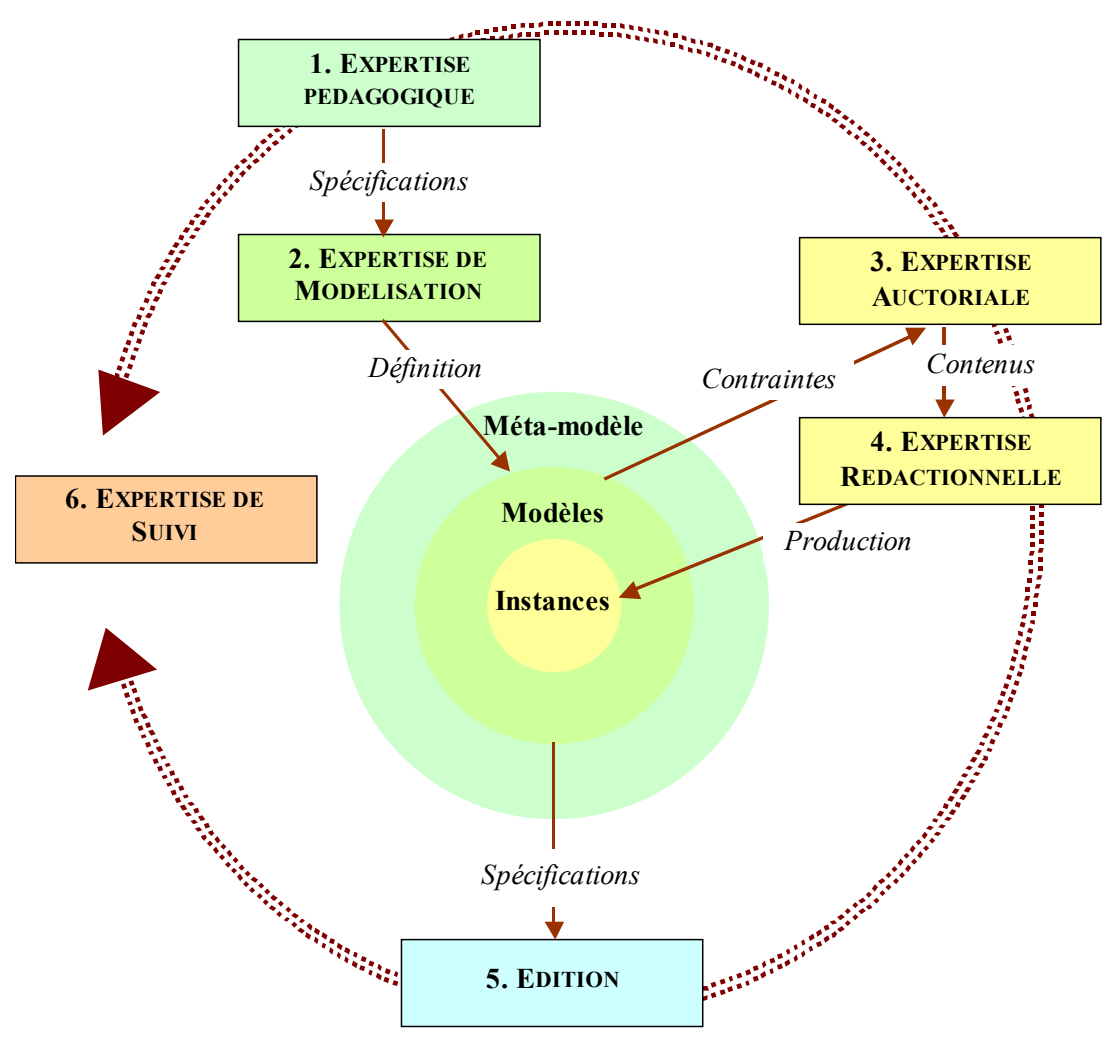

Figure 6. Chaîne de production adaptée au méta-modèle SP/UL

\section{L'expérience XF01}

\subsection{Contexte}

Cette première expérience concernait la réalisation d'un support pédagogique numérique pour l'enseignement des bases de l'algorithmique et de la programmation (cours NF01), à l'Université de Technologie de Compiègne, en Tronc Commun (cycle de préparation à la formation d'ingénieur, équivalent au DEUG). Son objectif était avant tout de valider les hypothèses fondatrices de structuration logique, de scénarisation séparée et de mise à disposition d'actions de réappropriation. Elle a permis d'autre part d'observer un usage de formation autour d'un support ainsi conçu. Elle ne s'est par contre pas attachée à valider d'éventuels choix technologiques.

Traditionnellement le cours NF01 concerne 150 à 250 étudiants. Le dispositif d'enseignement est classiquement composé d'une heure et demi de cours magistral en amphithéâtre, de deux heures de travaux dirigés en groupes réduits (24 étudiants) 
et d'une heure de travaux pratiques sur machines en demi-groupe (12 étudiants). Face aux problèmes, également classiques, rencontrés au cours de cet enseignement (tels que la fidélisation des étudiants au cours, la séparation entre théorie et pratique ou l'autonomisation des étudiants), certains aménagements ont été recherchés. Ainsi le cours magistral a tenté de laisser plus de place à l'interaction avec les étudiants, sans beaucoup de succès, l'effectif trop important étant peu adapté à ce modèle. C'est dans ce contexte que l'expérience a été menée, afin d'observer ce qu'un dispositif pédagogique innovant, centré autour d'un support numérique, pouvait apporter. Une fois le support réalisé, il a été utilisé durant deux semestres d'enseignement, à l'automne 1999 et au printemps 2000. 230 étudiants suivaient le cours NF01 lors du premier semestre et 130 lors du second. Dans les deux cas 20 étudiants ont été isolés (au hasard) pour suivre l'expérience (intitulée XF01), tandis que les autres continuaient de suivre le cours selon le schéma classique. Le dispositif pédagogique de XF01 était constitué de 3 heures par semaine de séances tutorées autour du support numérique, dans une salle informatisée, (en remplacement des cours et TD) et d'1 heure de travaux pratiques (identique au schéma classique).

La séance tutorée regroupait cours et exercices selon le déroulement suivant :

- Les étudiants préparent chez eux le cours et les exercices à partir d'un support papier

- Au début de chaque séance les étudiants posent des questions au tuteur afin de préciser les points mal compris

- Les étudiants travaillent en binôme de façon autonome avec le support numérique : lecture/annotation du cours et résolution des exercices (avec accès à des indices et aux solutions). Le tuteur répond à des questions des étudiants, reprend l'attention de l'ensemble de la classe pour exposer un point ardu, interroge des étudiants pour les guider, etc.

- Les étudiants participent à un ensemble de travaux de restitution (synthèse des questions et réponses posées, résolution d'exercices au tableau, réponse à des QCM).

\subsection{Mise en oeuvre}

L'expertise pédagogique a été menée selon la méthode inspirée des actes pédagogiques [GHI 01] et a permis de définir les modèles suivants :

\begin{tabular}{|c|l|l|l|}
\hline Modèles & \multicolumn{1}{|c|}{ Entête } & \multicolumn{1}{|c|}{ Corps } & \multicolumn{1}{c|}{ Actions } \\
\hline ULExposition & Mots-clés & $\begin{array}{l}\text { Définition } \\
\text { Exemple } \\
\text { Méthodologie } \\
\text { Illustration } \\
\text { Approfondissement }\end{array}$ & $\begin{array}{l}\text { Annoter } \\
\text { Mettre en valeur } \\
\text { Donner le sommaire }\end{array}$ \\
\hline ULPratique & $\begin{array}{l}\text { Difficulté } \\
\text { Temps }\end{array}$ & $\begin{array}{l}\text { Enoncé } \\
\text { Indices }\end{array}$ & $\begin{array}{l}\text { Résoudre } \\
\text { Regarder un indice }\end{array}$ \\
\hline
\end{tabular}




\begin{tabular}{|c|c|c|c|}
\hline Modèles & Entête & Corps & Actions \\
\hline & & Solution & $\begin{array}{l}\text { Regarder une } \\
\text { solution } \\
\text { S'auto évaluer }\end{array}$ \\
\hline ULDiscussion & & $\begin{array}{l}\text { Question } \\
\text { Réponse }\end{array}$ & $\begin{array}{l}\text { Regarder les } \\
\text { questions et } \\
\text { réponses déjà } \\
\text { posées }\end{array}$ \\
\hline ULEvaluation & & $\begin{array}{l}\text { Questions } \\
\text { Propositions de } \\
\text { réponses } \\
\text { Réponses correctes }\end{array}$ & $\begin{array}{l}\text { Répondre } \\
\text { Corriger ses } \\
\text { réponses } \\
\text { Etre évalué }\end{array}$ \\
\hline SPEtape & $\begin{array}{l}\text { Objectifs } \\
\text { Résumé }\end{array}$ & $\begin{array}{l}\text { Liste d'ULExposition } \\
\text { et ULPratique, puis } \\
\text { liste } \\
\text { d'ULDiscussion, puis } \\
\text { liste d'ULEvaluation }\end{array}$ & \\
\hline
\end{tabular}

Tableau 3. Modèles d'UL et de SP définis dans le cadre de XF01

L'implémentation des modèles de SP et UL a été réalisée à l'aide du SGBDR Microsoft Access. L'objectif de ce premier développement n'était pas de valider des choix technologiques, mais d'expérimenter les concepts du méta-modèle SP/UL. A ce titre le choix d'Access permettait un développement très rapide, notamment en ce qui concerne l'interface. Le schéma relationnel permet d'exprimer les modèles, avec deux tables (une pour les données statiques et une pour les données dynamiques) pour un modèle d'UL, et deux tables (une pour les méta-informations et une pour les scénarii) pour le modèle de SP d'étape.

La rédaction a été menée à partir du polycopié existant pour le cours de NF01, en structurant l'information pour qu'elle coïncide avec les champs préalablement définis dans le modèle relationnel.

Un prototype a ensuite été réalisé, grâce à l'éditeur de formulaires d'Access, afin de permettre la consultation des contenus et l'exécution des actions sur support numérique. Un support papier a également été produit, grâce à l'éditeur d'états d'Access, afin de rendre disponible l'ensemble des contenus spatiaux indépendamment d'un accès à un ordinateur. Enfin une partie des contenus, les questions et réponses des ULDiscussion, ont été édités en HTML et mis à disposition sur le Web. 
26 Nom de la Revue. Volume $\mathrm{X}-\mathrm{n}^{\circ} \mathrm{X} / 2000$

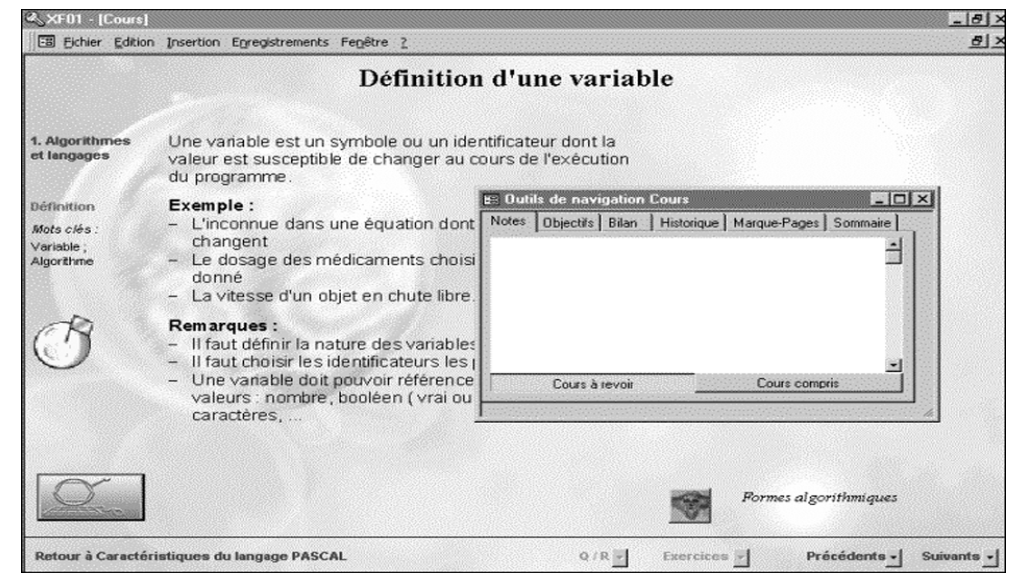

Figure 7. Version écran

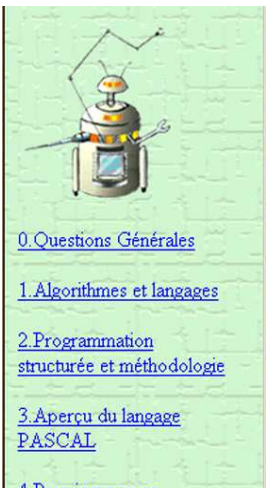

\section{Questions / Réponses}

ETAPE 1 : Algorithmes et langages

Question 1 : A quoi servent les diagrammes de Conway ?

Les diagrammes de Conway servent à représenter des langages.

Question 2: Pourquoi utilise-t-on des "ronds" et des "carrés" dans les diagramr

Pour distinguer les éléments terminaux et non-terminaux du langage. Les éléments terminal du langage, tandis que les éléments non-terminaux, inscrits dans un "carré", doivent être $d$

Dans le langage : $L=a-B-c$ (où a et $c$ sont terminaux et $B$ non-terminal), on sait qu'on.

Figure 8. Version Web

Dans le langage : $L=a-B-c$ (ou a et $c$ sont terminaux et $B$ non-termina), on sait qu'on

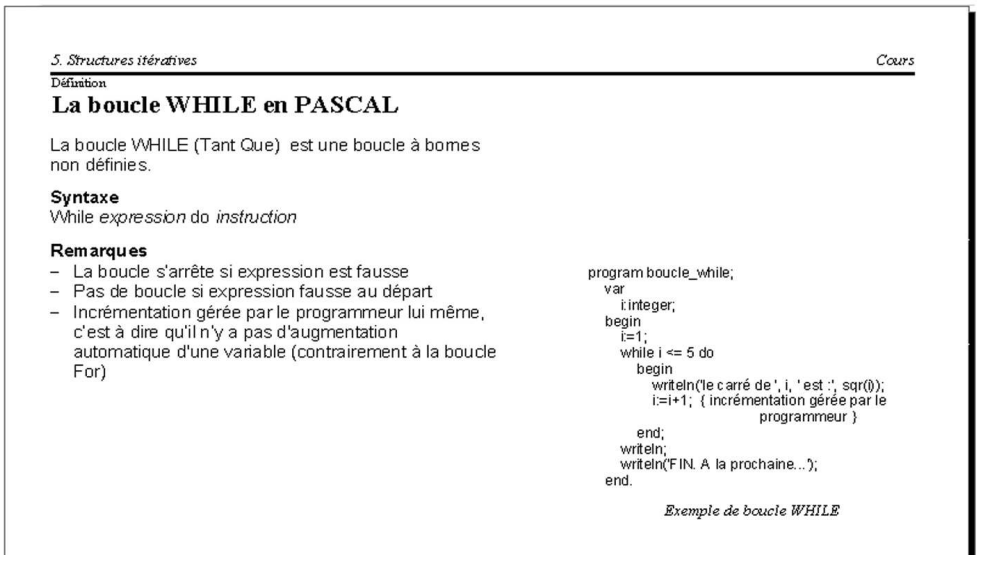

Figure 9. Version papier 


\subsection{Observations}

Cette expérience a permis de valider l'applicabilité du méta-modèle, dans le cadre particulièrement favorable où l'expert pédagogique est également l'expert du modèle. Elle a également permis les premières observations concernant le processus particulier de rédaction de contenus dans le cadre d'une structure imposée a priori par un modèle, la rigidité du schéma relationnel compliquant sensiblement cette tâche par rapport à une DTD. Nous avons mis en œuvre une approche d'édition multi-support, écran, papier et Internet, qui a notamment montré les limites du SGBRD dans ce cadre et conforté la solution XML. En effet, cette approche d'édition étant fondée sur la logique d'interface intégrée à Access, elle n'a pas permis une implémentation souple des FC, et est restée limitée aux possibilités classiques de la base de données en terme d'IHM.

Le support réalisé ayant été utilisé en conditions réelles d'enseignement durant deux semestres, il a également été l'occasion d'expérimenter l'usage. Quatre modes d'évaluation ont été menés aux cours des expériences, afin d'observer l'appréciation par les étudiants, l'appréciation par les tuteurs, la comparaison de résultats aux examens avec les étudiants suivant l'approche classique, et d'avoir l'avis d'un expert de l'évaluation des dispositifs pédagogiques (Franck Ghitalla en l'occurrence, le rapport lié à l'expérience étant disponible dans [GHI 01]). Le volume de l'expérience reste trop peu significatif pour en tirer des conclusions généralisables, nous avons néanmoins observé un taux d'acceptation de l'approche et du logiciel très élevé, et des résultats aux examens globalement meilleurs (on pourra se référer à [CRO 01] pour de plus amples précisions sur l'approche méthodologique et les résultats pédagogiques de l'expérience XF01).

\section{Le projet SCENARI}

\subsection{Contexte}

Le projet SCENARI (Système de Conception d'Enseignements Numériques Actifs Réutilisables et Interactifs) a pris naissance au début de l'année 2000 dans le cadre d'un partenariat entre le département de Formation Continue de l'UTC, l'entreprise Axa et les centres de formation de l'Association pour l'Enseignement des Assurances (AEA) et de l'Institut Polytechnique d'Enseignement Comtois (ICEP). L'objectif général du projet était la constitution d'un dispositif numérique de formation à distance pour les contenus de l'AEA et de l'ICEP, pour la formation continue au sein d'Axa, l'UTC étant le partenaire technologique ayant en charge le développement informatique. Fonctionnellement, le système devait permettre la production et la maintenance aisée de formations scénarisées, la personnalisation de ces formations à partir d'une prescription pédagogique et leur mise en oeuvre par édition des documents sur des supports papiers et numériques. Le volume des 
documents à traiter étant de l'ordre de plusieurs milliers de pages, le mode de conception devait répondre à une problématique de massification réelle. Concrètement le méta-modèle SP/UL était donc une réponse a priori adéquate au problème posé, et le projet SCENARI un terrain d'application adéquat pour une implémentation fidèle des concepts. Le projet a été mis en oeuvre à travers la réalisation de deux maquettes lors de l'été 2000 , permettant de finaliser les concepts et de démontrer les potentialités de l'approche, puis d'un prototype ayant vocation à prendre en charge l'ensemble des aspects du méta-modèle SP/UL.

\subsection{Mise en auvre}

La modélisation a été menée à partir des documents existants dans les centres de formation, et principalement à partir des documents papiers de formation à distance de l'AEA. Une étude approfondie de ces documents menée en collaboration entre des experts du contenu et des experts de la modélisation a permis d'extraire les structures logiques permettant de représenter les contenus :

\begin{tabular}{|c|c|c|c|c|}
\hline \multicolumn{5}{|c|}{ SP Module } \\
\hline \multicolumn{5}{|c|}{ SP sous-Module } \\
\hline \multicolumn{5}{|c|}{ SP Session } \\
\hline \multicolumn{5}{|c|}{ SP sous-Session } \\
\hline $\begin{array}{c}\text { SP Etape } \\
\text { Général }\end{array}$ & SP Etapes Cours & SP Etapes Exercice & $\begin{array}{c}\text { SP Etapes } \\
\text { Activité }\end{array}$ & SP Etapes QCM \\
\hline $\begin{array}{l}\text { UL Cours } \\
\text { Général } \\
\text { UL Synthèse } \\
\text { UL Exercice } \\
\text { Général }\end{array}$ & $\begin{array}{l}\text { UL Explication } \\
\text { UL Exemple } \\
\text { UL Bibliographie } \\
\text { UL Définition } \\
\text { UL Document } \\
\text { UL Méthodologie } \\
\text { UL Illustration }\end{array}$ & $\begin{array}{l}\text { UL Exercice } \\
\text { UL Exercice de } \\
\text { synthèse } \\
\text { UL Application de } \\
\text { Méthodologie }\end{array}$ & UL Activité & $\begin{array}{l}\text { UL Question à Choix } \\
\text { Multiple } \\
\text { UL Question Evaluable } \\
\text { Automatiquement }\end{array}$ \\
\hline
\end{tabular}

Tableau 4. Modèles d'UL et de SP définis dans le cadre AXA/AEA/ICEP

La première maquette réalisée avait pour objectif de proposer un système de formation on-line intégrant les contenus, ainsi que des actions de réappropriation et la gestion dynamique des productions des apprenants. Cette maquette a été programmée sur la base d'un compilateur permettant de générer du PHP à partir des documents XML (pour la partie statique) et des servlets Java (pour la partie dynamique). Notons que le compilateur n'utilisait pas la technologie XSL et que les servlets n'implémentait pas la notion de FC (mais un comportement programmé en dur).

Une seconde maquette a été réalisée, avec un double objectif : permettre une utilisation au sein d'un dispositif non connecté à Internet et démontrer les possibilités d'édition multi-support du modèle de conception. Elle a donc été réalisée à partir de la même base de contenu, dans un format 100\% HTML généré grâce à des programmes XSL. Cette maquette n'intégrait pas la dimension dynamique des SP et UL et ne proposait donc qu'une représentation statique des contenus. Elle a été 
utilisée lors d'une expérience menée par Axa, dans le cadre d'une formation continue de dix semaines concernant une quinzaine de collaboratrices d'agent dans le domaine de la santé. Le support a été testé pendant une demi-journée au cours d'une séance de regroupement en présentiel de deux jours avec formateur.

Enfin le prototype a été réalisé (par Sylvain Spinelli, responsable du développement dans le cadre du projet SCENARI) avec pour objectif d'être une implémentation fidèle du méta-modèle SP/UL, intégrant notamment complètement les principes de scénarisation (avec transitions et contraintes) et des unités dynamiques (avec gestion des productions, calcul des valeurs, etc.). Ce système est une application Web, fondée sur Java (Servlets et JSP). La puissance et l'originalité de cette application résident notamment dans son implémentation réelle des FC. L'exemple ci-dessous présente un exemple simplifié de FC et de l'interface obtenue.

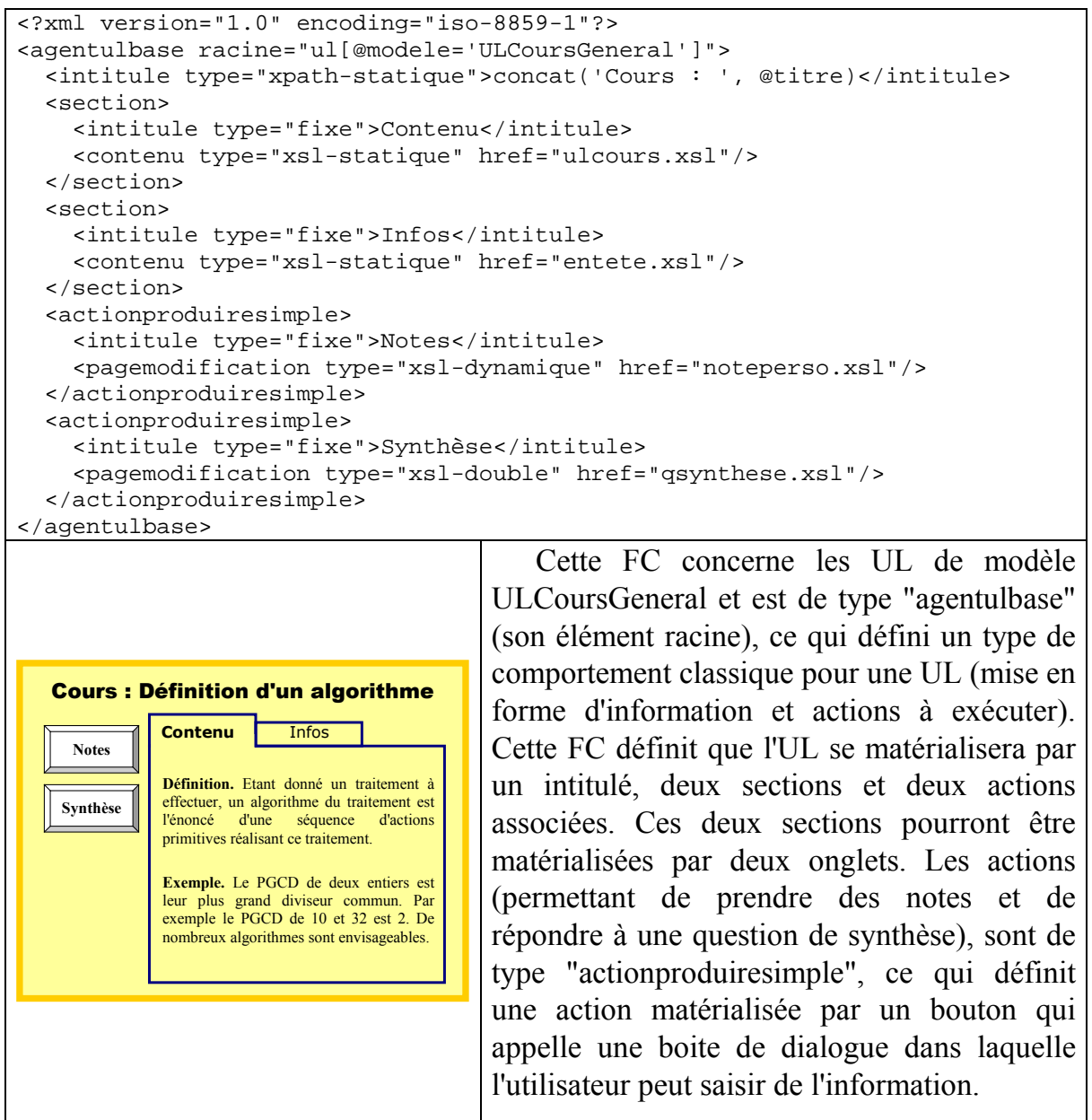

Tableau 5. Exemple simplifié 
L'intégration de cette notion permet à l'application de se définir comme un métaenvironnement de formation, c'est à dire un environnement générique paramétrable en fonction de modèles particuliers (le système n'est pas lié à des modèles pédagogiques a priori, mais suppose une définition de ces modèles et leur implémentation sous forme de FC pour chaque contexte).

En parallèle de ce prototype permettant l'implémentation de comportements complexes pour la consultation sur support numérique, nous avons enfin cherché à développer une édition papier d'une qualité propre à convenir aux besoins pratiques de l'AEA. En effet, indépendamment de la mise en place expérimentale et progressive de la formation en ligne, cela lui permettait d'intégrer plus rapidement notre système dans son dispositif de formation continue actuel papier-crayon. Nous avons choisi pour cette édition le langage $\mathrm{LaTeX}$, qui est actuellement une des solutions les plus puissantes et offrant la meilleure qualité de mise en page. L'édition est donc réalisée grâce à des XSL permettant de générer le LaTeX à partir du XML, puis avec un processeur TeX permettant d'en obtenir une version PDF (Les technologies XML propose une solution pour l'édition papier, le langage des Formatting Objects (FO). Néanmoins nous avons temporairement préféré LaTeX, les possibilités de FO étant encore moindres. Dans un soucis d'homogénéité technologique générale nous envisageons le passage à $\mathrm{FO}$ dans une version ultérieure de l'application).

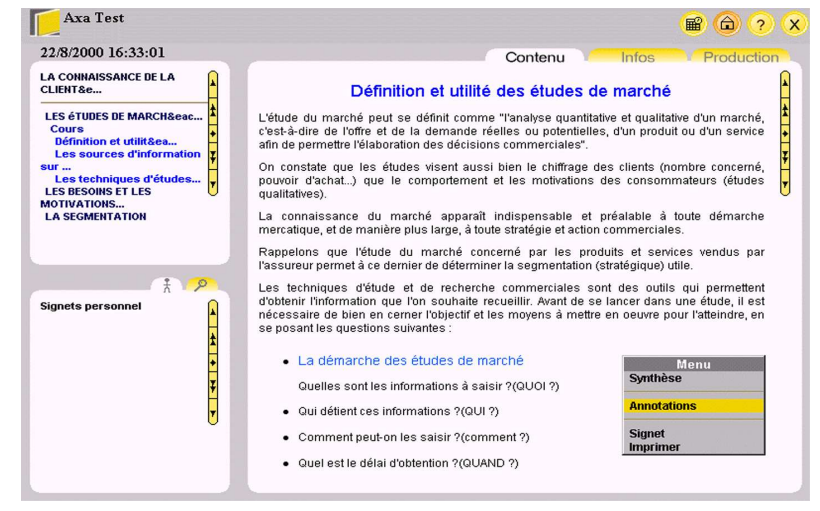

Figure 10. Première maquette 


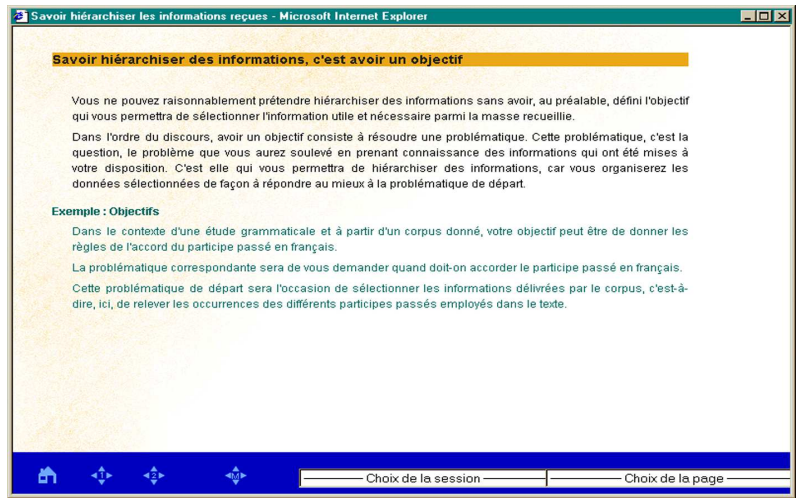

Figure 11. Seconde maquette

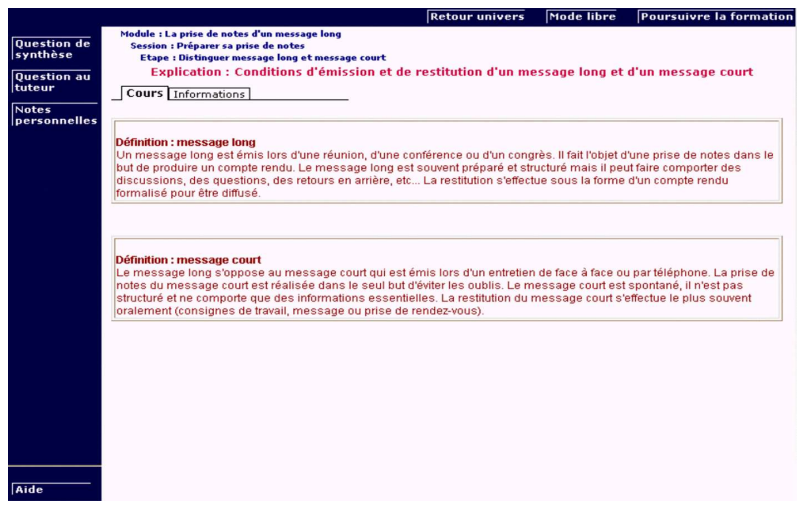

Figure 12. Prototype

Chapitre 1

Distinguer message long ct message court

1 Conditions d'emission et de restitution d'un message long el d'un message court

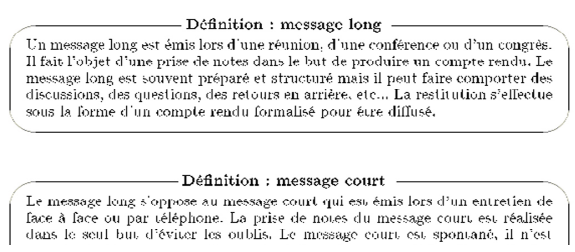

Figure 13. Version papier 


\subsection{Observations}

Le projet SCENARI a été le terrain qui a réellement permis la confrontation du modèle et de la réalité. Le méta-modèle repose sur l'hypothèse que des modèles pédagogiques permettent de contextualiser le modèle documentaire. Nos expériences ont montré qu'il était possible de réaliser de tels modèles, avec diverses méthodes permettant d'expliciter une pratique, préalablement inscrite dans des documents ou non.

Les modèles ainsi définis ont pu être exploités pour mettre en oeuvre une rédaction de contenus et scénarii structurés logiquement. Après diverses tentatives nous avons mis au point une approche méthodologique permettant la rédaction des contenus dans le respect des contraintes de structuration imposées par les modèles, en séparant les fonctions d'auteur et rédacteur XML. La formalisation des contraintes liées au modèle dans une procédure s'est révélée indispensable pour obtenir une rédaction efficiente et de qualité. Nous avons également mis en exergue la relativité qui existe dans le niveau de granularisation des contenus, afin de gérer l'équilibre entre des contenus finement structurés mais difficiles à produire et des contenus plus grossièrement représentés et plus difficiles à exploiter automatiquement dans une logique de réutilisation (sur des supports différents notamment).

Nos expériences d'édition multi-support, à travers des supports numériques offline et on-line et des supports papiers ont montré l'efficience de l'approche et son intérêt dans un cadre de massification. La structuration logique des contenus permet en effet de garantir a priori que les contenus pourront être exploités indépendamment d'un cahier des charges initial et d'une technologie particulière d'édition. L'investissement lourd consenti pour la production de contenus est de ce fait rationalisé en leur assurant une pérennité incomparablement supérieure à celle d'une technologie de mise en forme, à la durée de vie généralement très faible. A titre d'exemple le passage de la première à la seconde maquette, a été réalisé pour un coût de travail d'environ quatre heures (de programmation XSL), pour un volume de l'ordre de mille pages de contenu, soit environ 15 secondes par page. Aucune autre approche que la structuration logique n'aurait permis un tel changement de support à un coût aussi réduit.

Les premières implémentations des FC qui avaient été menée dans le cadre de XF01 et des maquettes SCENARI relevaient d'approches classiques, ne permettant pas réellement d'envisager l'édition d'interfaces interactives complexes dans une logique similaire à celle des feuilles de styles. Le prototype SCENARI a permis de prouver la faisabilité d'une telle approche, l'enjeu est à présent de stabiliser le langage de FC et de l'expérimenter sur de nouvelles applications. En juin 2002 le moteur SCENARI aura permis de créer trois applications pédagogiques différentes (avec des jeux de FC différents) dans le cadre du projet AXA/AEA/ICEP : une application orientée centre de formation, une application orienté auto-formation en entreprise et une application orientée révision en centre ressource. 


\section{Conclusion}

La démarche de conception que nous avons élaborée et expérimentée est basée sur une hypothèse documentaire permettant de répondre à la problématique de massification induite par les demandes croissantes en terme de formation et sur une hypothèse pédagogique permettant de maintenir la contextualisation nécessaire à toute pratique d'apprentissage. Le méta-modèle SP/UL instrumentant cette démarche est une réponse qui se fonde sur la nature même du support, le calcul dynamique, et en exploite les propriétés pour permettre une approche industrielle de conception. L'objet de notre travail est destiné à être exploité par des pédagogues et des experts du contenu afin qu'ils puissent mettre en oeuvre leurs expertises dans le cadre de la conception d'un support numérique. En d'autres termes le modèle est méta car il n'apporte pas l'expertise pédagogique, mais uniquement un cadre conceptuel pour exprimer une telle expertise pour chaque contexte particulier. Si notre recherche s'inscrit dans le domaine de l'informatique, elle ne peut-être dissociée de la pratique qui la sous-tend. Or cette pratique est en genèse, et la relation entre pratique et technique étant à double sens, il n'est pas possible de valider complètement notre proposition sans une pratique ad hoc, ni possible de mettre en place la pratique sans la technique correspondante. Seul le renouvellement d'expériences de plus en plus larges et de plus en plus diverses permettra réellement de montrer la validité de l'approche que nous proposons. Nous pouvons néanmoins poser dès à présent que nos concepts fondateurs se sont révélés efficients, quitte à ce que nos travaux futurs nous amènent à en réviser la mise en oeuvre sous certains aspects technologiques.

Les projets XF01 et SCENARI ont fourni un cadre concret d'application pour nos travaux. Ceci est fondamental dans la mesure où un modèle de conception ne peut-être pensé en dehors d'un usage réel. Ces expériences ont avant tout permis d'affiner le méta-modèle SP/UL pour aboutir à un résultat conforme avec une pratique de conception portant sur plusieurs milliers de pages de contenus. Ces expériences ont donc permis la mise au point des choix méthodologiques, mais également des choix technologiques, puisque l'usage d'XML s'est imposé, après diverses tentatives préalables, comme la solution la plus adaptée, par sa puissance et sa souplesse. L'extension apportée avec les FC a également pu être mise en oeuvre permettant réellement d'envisager une approche documentaire respectant les contraintes de la pédagogie (l'interactivité). En effet une solution générique de modélisation logique est inutile si elle n'est pas associée à une solution aussi générique de présentation physique. Les FC permettent de répondent à ce problème grâce à un langage permettant de spécifier et grâce à des technologies permettant de réaliser automatiquement des interfaces homme-machine interactives.

Si nos expériences ont permis de concrétiser l'ensemble des concepts du métamodèle SP/UL, certaines de ces mises en oeuvre n'ont pas été suffisamment expérimentées pour être complètement validées. Nous avons cherché à élaborer notre approche tout en expérimentant l'usage, en ayant néanmoins conscience que certains de nos concepts précédaient l'usage et donc ne seraient validés ou invalidés 
qu'après qu'une pratique eût tenté de se les réapproprier. Il en est par exemple ainsi des scénarii pédagogiques, qui n'ont pu être produits à grande échelle, les auteurs n'ayant pas encore de besoins réels car n'ayant pas encore de contextes pédagogiques pour les mettre en oeuvre. Notre approche a également montré que la réutilisation des SP et UL, pour un changement de contexte pédagogique ou technologique, était possible techniquement. Néanmoins aucune pratique n'existe réellement encore pour valider cette possibilité. Mais de nombreuses questions demeurent quant aux possibilités effectives de rédiger des contenus réutilisables, quant à la part des contenus réutilisables par rapport aux contenus exclusivement contextuels ou quant au coût nécessaire pour produire de tels contenus génériques. Seule une pratique de grande envergure montrera à terme s'il est effectivement rentable de produire des contenus nécessaires et suffisants pour les mobiliser dans différents contextes.

Nos travaux nous amènent aujourd'hui à considérer deux ouvertures majeures pour le futur. La première est la finalisation d'une application techniquement stable à partir de notre prototype actuel afin de la proposer à d'autres utilisateurs. La demande étant en pleine croissance pour de tels systèmes offrant des solutions pour la conception rationalisée de volumes importants de formation, notre système devrait trouver une place sur ce marché. La seconde est le recherche de solutions plus poussées pour l'intégration de contenus audiovisuels et interactifs. Ce type de contenu n'existe encore que très rarement à une échelle massive dans le domaine de la formation, néanmoins la démocratisation du numérique nous laisse imaginer qu'ils vont prendre une place de plus en plus importante dans la pratique. Il nous sera donc nécessaire de prolonger nos travaux afin d'être en mesure de les intégrer avec les mêmes avantages que ceux que nous avons permis pour les documents spatiaux.

\section{Bibliographie}

[AJH 00] AJHOUN R, BENKIRAN A, "SMART-Learning: Adaptative telelearning system on the web", actes du colloque TICE'2000, Troyes, France, pp.23-29, octobre 2000.

[BAC 94] BACHIMONT B, "Le contrôle dans les systèmes à base de connaissance : Contribution à l'épistémologie de l'intelligence artificielle", édition Hermes, 1994.

[BAC 96] BACHIMONT B, "Herméneutique matérielle et artéfacture : des machines qui pensent aux machines qui donnent à penser", mémoire de Thèse en épistémologie, édition Ecole Polytechnique, 1996.

[BAC 98] BAChimont B, Charlet J, "PolyTex : un environnement pour l'édition structurée de polycopiés électroniques multisupports", actes du colloque EuroTex'98, France, 1998.

[BAC 99] BACHIMONT B, "Intelligence artificielle et écriture dynamique : De la raison graphique à la raison computationnelle", in Au nom du sens coordonné par Jean Petitot, édition Grasset, 1999.

[BAL 96] Balpe J, Lelu A, PApy F, Saleh I, "Techniques avancées pour l'hypertexte", édition Hermes, 1996. 
[BAS 97] BASTIEN C, SCAPIN D, "Ergonomic criteria for evaluating the ergonomic quality of interactive systems", Behaviour and Information Technology, n¹6, pp.226-231, 1997.

[BOUL 00] BOULLIER D, "La loi du support : Leçons de trois ans d'enseignement numérique à distance", Les Cahiers du Numérique, n², vol.1, pp.145-172, édition Hermes, 2000.

[BOUR 00] Bourda Y, Helier M, "Métadonnées et XML : applications aux "objets pédagogiques"', actes du colloque TICE'2000, Troyes, France, pp.135-141, octobre 2000.

[BRU 97] BRUILlaRd E, "Les machines à enseigner", édition Hermes, 1997.

[CHA 96] Chalvin D, CoQuerelle J, "Encyclopédie des pédagogies de formation : Histoire et principales approches", édition ESF, 1996.

[CHO 00] Choplin H, Degrugillier D, Galisson A, Morin S, "Comment réaliser un simulateur pédagogique ? : Un exemple conçu et développé par le groupe des écoles de télécommunications", actes du colloque TICE'2000, Troyes, France, pp.333-341, octobre 2000 .

[CRO 99] CROZAT S, Hu O, TRIGANo P, "EMPI : Une méthode informatisée pour l'évaluation des didacticiels multimédias", Revue de l'Interaction Homme Machine, n², vol.1, édition Hermes, 1999.

[CRO 01] CROZAT S, TRIgano P, "Conception de supports hypermédias pour une exploitation pédagogique : modèle et expérience", Revue Spirale, à paraître (octobre 2001).

[FOR 97] Forte E, Wentland-Forte M, Duval E, "The ARIADNE project, (part I) : Knowledge pools for computer based and telematics supported classical and open education", European Journal of Engineering Eduvation, $n^{\circ} 1$, vol.22, pp.61-74, mars 1997.

[FOR 97] FORTE E, Wentland-Forte M, Duval E, "The ARIADNE project, (part II) : Knowledge pools for computer based and telematics supported classical and open education", European Journal of Engineering Eduvation, n¹, vol.22, pp.153-166, mars 1997.

[For 99] Forte E, Haenni F, Warkentyne K, Duval E, Cardinaels K, Vervaet E, Hendrikx K, Wentland-Forte M, Simillion F, "Semantic and pedagogic interoperability mechanisms in the ARIADNE educational repository", ACM SIGMOD, $\mathrm{n}^{\circ} 1$, vol.28, pp.20-25, 1999.

[GAS 98] GASNERAu P, "Serveur de Didacticiels Multimédia", actes du colloque NTICF'98, Rouen, novembre 1998.

[GHI 01] GHITALla F, "Les formats pédagogiques : Théorie expérimentale de l'interaction et de la scénarisation pédagogique", mémoire de Recherche du pôle NTE de la région Picardie, édition Université de Technologie de Compiègne, 2001.

[GIL 00] GIL P, "e-formation : NTIC et reengineering de la formation professionnelle", édition Dunod, 2000.

[GOO 79] Goody J, "La raison graphique : La domestication de la pensée sauvage", édition Les éditions de minuit, 1979. 
36 Nom de la Revue. Volume X $-\mathrm{n}^{\circ} \mathrm{X} / 2000$

[GUE 99] Guegot F, Necaille C, "Modélisation d'hyper documents dans un système hypermédia pour la formation", in Hypertextes, hypermédias et internet coordonné par Jean-Pierre Balpe, pp.73-87, édition Hermes, 1999.

[HU 00] Hu O, Crozat S, Trigano P, " Evaluer les logiciels multimédias pédagogiques : objectivité versus subjectivité et généricité versus contextualité ", RES ACADEMICA (Revue de l'Enseignement Supérieur), Vol.17, $\mathrm{N}^{\circ} 1$ et 2, 2000.

[HU 01a] Hu O, "Contribution à l'évaluation des logiciels multimédias pédagogiques", mémoire de Thèse en Informatique, édition Université de Technologie de Compiègne, à paraître (2001).

[HU 01b] Hu O, Trigano P, Crozat S, "Une aide à l'évaluation des logiciels multimédias de formation", Sciences et Techniques Educatives, édition Hermès, à paraître (2001).

[ISA 00] Isabelle C, Nkambou R, Dufresne A, "Système interactif et hypermédia pour l'intégration de technologie de l'information chez des futurs enseignants", actes du colloque TICE'2000, Troyes, France, pp.169-177, octobre 2000.

[LIN 96] LiNARD M, "Des machines et des hommes : Apprendre avec les nouvelles technologies", édition L'Harmattan, 1996.

[MED 90] MEDA J, "Evaluer les logiciels de formation", édition Les Editions d'Organisation, 1990.

[MIC 97] MichARD A, "XML, langage et applications", édition Eyrolles, 1997.

[MOU 00] Moulin C, Pazzaglia C, "Création dynamique d'activités adaptées dans un environnement d'apprentissage à distance", actes du colloque TICE'2000, Troyes, France, pp.179-190, octobre 2000 .

[PAS 01] DE LA PASSARdiere B, Giroire H, "XML au service des applications pédagogiques", revue STE, ${ }^{\circ} 1$, vol.8, édition Hermès, 2001.

[PEC 00] PeCCOud F, "Les nouvelles pratiques de formation", Les Cahiers du Numérique, n², vol.1, pp.89-102, édition Hermes, 2000.

[PER 00] Perava D, "Le cyberespace : Un dispositif de communication et de formation médiatisé", édition DeBoeck Université, 2000.

[PIA 67] Piaget J, "La psychologie de l'intelligence", édition Armand Collin, 1967.

[REV 00] REVERCHON A, "Les universités françaises lancent le chantier de l'enseignement à distance", édition Le monde économie, 1er février 2000.

[STI 94] STIEgLer B, "La technique et le temps, Tome I : La faute d'Epiméthée", édition Galilée, 1994.

[STI 94] Stiegler B, "La technique et le temps, Tome II : La désorientation", édition Galilée, 1994.

[WEI 99] WeISSBerg J, "Entre production et réception: l'hypermédiation, une mutation des savoirs symboliques", in Hypertextes, hypermédias et internet coordonné par Jean-Pierre Balpe, pp.73-87, édition Hermes, 1999. 
[ZEI 99] Zeiliger R, Belisle C, Cerratto T, "Implementing a constructivist approach to web navigation swupport", actes du colloque ED-MEDIA'99, Etats-Unis, édition Eds Collis, juin 1999.

Stéphane Crozat, ingénieur en informatique, a travaillé dans le cadre de sa thèse au sein du laboratoire Heudiasyc sur l'évaluation, puis la conception et l'expérimentation de logiciels pédagogiques. Il soutiendra sa thèse de doctorat, intitulée "Eléments pour la conception industrialisée des supports pédagogiques numériques" le 22 mars 2002. Il travaille actuellement à la Formation Continue de l'UTC au développement de produits pédagogiques pour les entreprises.

Philippe Trigano est professeur des universités, en informatique, à l'Université de Technologie de Compiègne. Il dirige l'équipe ROC (réseaux, optimisation, communication), l'un des trois themes de recherche du laboratoire Heudiasyc (UMR CNRS 6599). Il travaille dans le domaine de l'intelligence artificielle et du multimédia, appliques à la formation. 